\title{
Approximation of Coupled Stokes-Darcy Flow in an Axisymmetric Domain
}

\author{
V.J. Ervin * \\ Department of Mathematical Sciences \\ Clemson University \\ Clemson, SC, 29634-0975 \\ USA.
}

January 20, 2013

\begin{abstract}
In this article we investigate the numerical approximation of coupled Stokes and Darcy fluid flow equations in an axisymmetric domain. The fluid flow is assumed to be axisymmetric. Rewriting the problem in cylindrical coordinates reduces the 3-D problem to a problem in 2-D. This reduction to 2-D requires the numerical analysis to be studied in suitably weighted Hilbert spaces. In this setting we show that the proposed approximation scheme has a unique solution, and derive corresponding a priori error estimate. Computations for an example with a know solution are presented which support the a priori error estimate. Computations are also given for a model of fluid flow in the eye.
\end{abstract}

Key words. axisymmetric flow; Stokes equation, Darcy equation, coupled fluid flow

AMS Mathematics subject classifications. 65N30

\section{Introduction}

For the past several years the investigation of the numerical approximation of coupled Stokes-Darcy fluid flow problems has been an active area of research. A number of different formulations have been studied. An approach introduced and analyzed by Layton, Schieweck, and Yotov in [27] formulates the problem in terms of the unknown velocity-pressure variables in both the Stokes and Darcy domains. Other researchers who have used this approach include [37, 8, 19, 17, 20]. In [14] Discacciati, Miglio, and Quateroni formulated the problem in terms of velocity-pressure unknowns in the Stokes domain and a pressure unknown (satisfying the Poisson equation) in the Darcy domain. Other researchers who have used this approach include [30, 21, 11, 9]. Another approach is to use the Brinkman equations for the coupled problem [22]. In this approach the Stokes modeling equations and the Darcy equations are melded together using cutoff functions to obtain a single modeling

*email: vjervin@clemson.edu. 
equation throughout the coupled domain. Though computationally attractive this approach lacks rigorous mathematical analysis as to the relationship between the computed approximation and the true solution.

In this paper we investigate the special case of a 3-D Stokes-Darcy fluid flow problem in an axisymmetric domain, having an axisymmetric solution. Our motivation for considering this problem was to model fluid flow in the eye. Using the axisymmetric, we reformulate the problem in cylindrical coordinates, reducing the 3 -D problem in $(x, y, z)$ to a 2 -D problem in $(r, z)$. Accompanying this reduction in spatial dimension is that the function space setting for the problem is now cast in weighted Sobolev spaces.

The numerical analysis of the finite element approximation to the axisymmetric Stokes problem was presented in [4]. (See also [5, 29, 16, 6].) For the axisymmetric Darcy problem the numerical analysis of the finite element approximation was recently given in [15]. Herein we combine the analysis of the axisymmetric Stokes and Darcy problems with the framework of [27] to obtain and analysis an approximation scheme for the coupled, axisymmetric Stokes-Darcy fluid flow problem.

This paper is organized as follows. In Section 2 the modeling equations are presented, rewritten in cylindrical coordinates, and a corresponding weak formulation for the solution derived. Following, in Section 3 we present the framework for the finite element approximation, establish existence and uniqueness of the finite element approximation, and derive an a priori error estimate for the approximation. Two examples are given in the Numerical Experiments section. The first example investigates the a priori error estimate for several different choices of approximating elements. The second example numerically investigates a model for fluid flow in the eye.

\section{Modeling Equations}

Let $\breve{\Omega} \subset \mathbb{R}^{3}$, denote the flow domain of interest. Additionally, let $\breve{\Omega}_{f}$ and $\breve{\Omega}_{p}$ denote bounded convex polygonal domains for the Stokes flow and Darcy flow, respectively. The interface boundary between the domains is denoted by $\breve{\Gamma}:=\partial \breve{\Omega}_{f} \cap \partial \breve{\Omega}_{p}$. Note that $\breve{\Omega}:=\breve{\Omega}_{f} \cup \breve{\Omega}_{p} \cup \breve{\Gamma}$. The outward pointing unit normal vectors to $\breve{\Omega}_{f}$ and $\breve{\Omega}_{p}$ are denoted $\breve{\mathbf{n}}_{f}$ and $\breve{\mathbf{n}}_{p}$, respectively. On $\breve{\Gamma}$ let $\breve{\mathbf{t}}_{1}, \breve{\mathbf{t}}_{2}$ denote linearly independent unit tangent vectors. We assume that there is an inflow boundary $\breve{\Gamma}_{i n}$, a subset of $\partial \breve{\Omega}_{f} \backslash \breve{\Gamma}$, which is separated from $\breve{\Gamma}$, and an outflow boundary $\breve{\Gamma}_{\text {out }}$, a subset of $\partial \breve{\Omega}_{p} \backslash \breve{\Gamma}$, which is also separated from $\breve{\Gamma}$. See Figure 2.1 for an illustration of the domain of the problem.

Define $\breve{\Gamma}_{f}:=\partial \breve{\Omega}_{f} \backslash\left(\breve{\Gamma} \cup \breve{\Gamma}_{\text {in }}\right)$, and $\breve{\Gamma}_{p}:=\partial \breve{\Omega}_{p} \backslash\left(\breve{\Gamma} \cup \breve{\Gamma}_{\text {out }}\right)$.

We assume that the flow in the porous domain $\breve{\Omega}_{p}$ is governed by the Darcy's equation subject to incompressibility of the fluid, a specified flow rate $(\Xi)$ across $\breve{\Gamma}_{\text {out }}$, and a non-penetration condition on $\breve{\Gamma}_{p}$. 

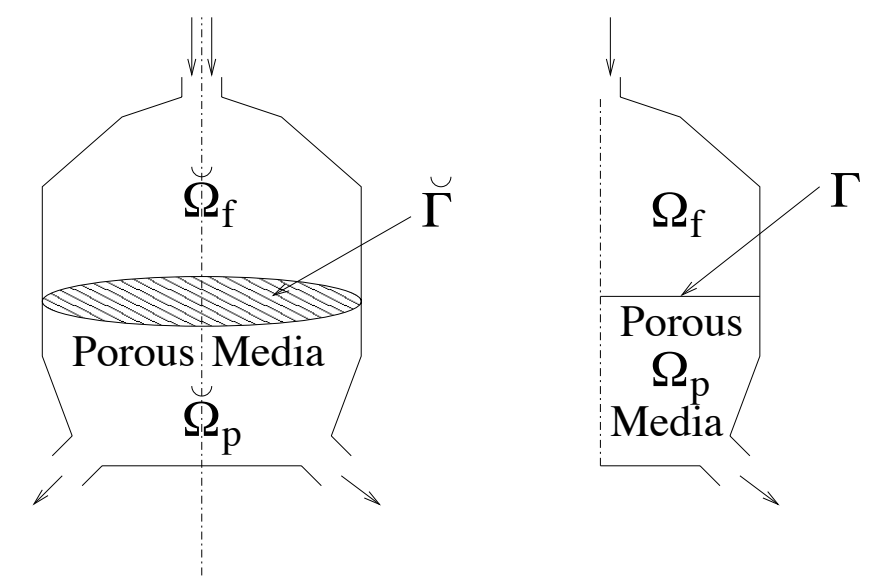

Figure 2.1: Illustration of axisymmetric flow domain.

For the Stokes flow:

$$
\begin{aligned}
-\nabla \cdot\left(2 \nu \breve{\mathbf{d}}\left(\breve{\mathbf{u}}_{f}\right)-\breve{p}_{f} \mathbf{I}\right) & =\breve{\mathbf{f}}_{f} \quad \text { in } \breve{\Omega}_{f}, \\
\nabla \cdot \breve{\mathbf{u}}_{f} & =0 \text { in } \breve{\Omega}_{f}, \\
\int_{\breve{\Gamma}_{i n}} \breve{\mathbf{u}}_{f} \cdot \breve{\mathbf{n}}_{f} d s & =-\Xi, \\
\breve{\mathbf{u}}_{f}=\mathbf{0} & \text { on } \breve{\Gamma}_{f} .
\end{aligned}
$$

where $\breve{\mathbf{u}}_{f}=\left[\begin{array}{l}u_{f x} \\ u_{f y} \\ u_{f z}\end{array}\right]=u_{f x} \mathbf{e}_{x}+u_{f y} \mathbf{e}_{y}+u_{f z} \mathbf{e}_{z}$, for $\mathbf{e}_{x}, \mathbf{e}_{y}, \mathbf{e}_{z}$ denoting unit vectors in the $x, y$ and $z$ directions, respectively, and $\breve{\mathbf{d}}(\breve{\mathbf{u}}):=1 / 2\left(\nabla \breve{\mathbf{u}}+(\nabla \breve{\mathbf{u}})^{T}\right)$ represents the deformation tensor. In (2.1)-(2.4) $\breve{\mathbf{u}}_{f}$ denotes the fluid's velocity, $\breve{p}_{f}$ the pressure, $\breve{f}_{f}$ an external forcing function, $\nu$ the fluid kinematic viscosity, and $\Xi$ a specified inflow rate for the fluid.

For the porous domain $\breve{\Omega}_{p}$ :

$$
\begin{aligned}
\nu_{\text {eff }} \breve{\mathbf{K}}^{-1} \breve{\mathbf{u}}_{p}+\nabla \breve{p}_{p} & =\breve{\mathbf{f}}_{p} \quad \text { in } \breve{\Omega}_{p}, \\
\nabla \cdot \breve{\mathbf{u}}_{p} & =0 \quad \text { in } \breve{\Omega}_{p}, \\
\int_{\breve{\Gamma}_{\text {out }}} \breve{\mathbf{u}}_{p} \cdot \breve{\mathbf{n}}_{p} d s & =\Xi \\
\breve{\mathbf{u}}_{p} \cdot \breve{\mathbf{n}}_{p}=0 & \text { on } \breve{\Gamma}_{p} .
\end{aligned}
$$

In (2.5)-(2.8) $\breve{\mathbf{u}}_{p}, \breve{p}_{p}, \breve{\mathbf{f}}_{p}$, denote the fluid velocity, pressure and external forcing functions, respectively. Additionally, in (2.5) $\nu_{\text {eff }}$ represents an effective kinematic fluid viscosity, and $\breve{\mathbf{K}}$ the permeability (symmetric, positive definite) tensor of the domain. For simplicity, we assume that there is a scalar function $\kappa$ such that $\kappa \mathbf{I}=\nu_{\text {eff }} \breve{\mathbf{K}}^{-1}$.

Across the interface $\breve{\Gamma}$ the flows are coupled via the conservation of mass and balance of normal forces. In addition, the Beavers-Joseph-Saffman (BJS) condition $[3,23,38]$ is used for the tangential 
forces boundary condition on $\breve{\Gamma}$.

$$
\begin{array}{r}
\breve{\mathbf{u}}_{f} \cdot \breve{\mathbf{n}}_{f}+\breve{\mathbf{u}}_{p} \cdot \breve{\mathbf{n}}_{p}=0, \quad p_{f}-\left(2 \nu \breve{\mathbf{d}}\left(\breve{\mathbf{u}}_{f}\right) \cdot \breve{\mathbf{n}}_{f}\right) \cdot \breve{\mathbf{n}}_{f}=p_{p} \quad \text { on } \breve{\Gamma}, \\
\breve{\mathbf{u}}_{f} \cdot \breve{\mathbf{t}}_{l}=-\alpha_{l}\left(2 \nu \breve{\mathbf{d}}\left(\breve{\mathbf{u}}_{f}\right) \cdot \breve{\mathbf{n}}_{f}\right) \cdot \breve{\mathbf{t}}_{l} \quad \text { on } \breve{\Gamma}, \quad l=1,2,
\end{array}
$$

where $\alpha_{1}, \alpha_{2}$ denote friction constants.

The boundary conditions (2.3) and (2.7) are commonly referred to as defective boundary conditions, as they do not uniquely define a solution to (2.1)-(2.8). In Section 2.1 we present a weak formulation of (2.1)-(2.8) and discuss the existence and uniqueness of the weak formulation. At the end of Section 2.1 we comment that, in addition to (2.3) and (2.7), the weak formulation implicitly imposes additional boundary conditions for $\breve{\mathbf{u}}_{f}$ on $\breve{\Gamma}_{\text {in }}$ and for $\breve{\mathbf{u}}_{p}$ on $\breve{\Gamma}_{\text {out }}$.

\subsection{Function Spaces and Weak Formulation}

In this section we introduce the function spaces needed to define the weak formulation for the coupled fluid flow problem described above.

Let $\breve{\Theta}:=\Theta \times[0,2 \pi) \subset \mathbb{R}^{3}$ be a bounded domain formed by revolving the polygon $\Theta$ around the $z$-axis. For the axisymmetric formulation we introduce the following weighted function spaces and associated norms. For any real $\alpha$ and $1 \leq p<\infty$, the space ${ }_{\alpha} L^{p}(\Theta)$ is defined as the set of measurable functions $w$ such that

$$
\|w\|_{\alpha} L^{p}(\Theta)=\left(\int_{\Theta}|w|^{p} r^{\alpha} d \mathbf{x}\right)^{1 / p}<\infty
$$

where $r=r(\mathbf{x})$ is the radial coordinate of $\mathbf{x}$, i.e. the distance of a point $\mathbf{x}$ in $\Theta$ from the symmetry axis. The subspace ${ }_{1} L_{0}^{2}(\Theta)$ of ${ }_{1} L^{2}(\Theta)$ denotes the functions $q$ with weighted integral equal to zero, $\int_{\Theta} q r d \mathbf{x}=0$.

We define the weighted Sobolev space ${ }_{1} W^{l, p}(\Theta)$ as the space of functions in ${ }_{1} L^{p}(\Theta)$ such that their partial derivatives of order less that or equal to $l$ belong to ${ }_{1} L^{p}(\Theta)$. Associated with ${ }_{1} W^{l, p}(\Theta)$ is the semi-norm $|\cdot|_{1 W^{l, p}(\Theta)}$ and norm $\|\cdot\|_{1 W^{l, p}(\Theta)}$ defined by

$$
|w|_{1 W^{l, p}(\Theta)}=\left(\sum_{k=0}^{l}\left\|\partial_{r}^{k} \partial_{z}^{l-k} w\right\|_{1 L^{p}(\Theta)}^{p}\right)^{1 / p}, \quad\|w\|_{1 W^{l, p}(\Theta)}=\left(\sum_{k=0}^{l}|w|_{1 W^{k, p}(\Theta)}^{p}\right)^{1 / p} .
$$

When $p=2$, we denote ${ }_{1} W^{l, 2}(\Theta)$ as ${ }_{1} H^{l}(\Theta)$.

For a domain $\Upsilon \subset \mathbb{R}^{3}$ we use the usual definitions and notation for Sobolev spaces. For vector functions $\mathbf{v}$ defined on $\Upsilon$ expressed in cylindrical coordinates we use the notation $\mathbf{v}(r, \theta, z)=$ $v_{r} \mathbf{e}_{r}+v_{\theta} \mathbf{e}_{\theta}+\mathbf{v}_{z} \mathbf{e}_{z}=\left(v_{r}, v_{\theta}, v_{z}\right)$, where $\mathbf{e}_{r}, \mathbf{e}_{\theta}, \mathbf{e}_{z}$ denote unit vectors in the $r, \theta$, and $z$ directions, respectively. Additionally, for $\mathbf{v}(r, z)$ defined on $\Theta$ we use $\mathbf{v}(r, z)=\left(v_{r}, v_{z}\right)=v_{r} \mathbf{e}_{r}+\mathbf{v}_{z} \mathbf{e}_{z}$.

Let $R_{\phi}$ denote a rotation with respect to $\phi$ about the $z$-axis. A function $\breve{v}$ is axisymmetric if $\breve{v}=\breve{v} \circ R_{\phi}$ for all $\phi \in(0,2 \pi)$. A vector function $\breve{\mathbf{v}}$ is rotationally invariant if $\breve{\mathbf{v}}=R_{-\phi} \circ \breve{\mathbf{v}} \circ$ $R_{\phi}$ for all $\phi \in(0,2 \pi)$. Let $\breve{H}^{s}(\breve{\Theta}) \subset H^{s}(\breve{\Theta}), s=0,1,2$ denote those functions in $H^{s}(\breve{\Theta})$ which are axisymmetric, and $\breve{H}^{s}(\breve{\Theta})^{3}$ the space of rotationally invariant $H^{s}(\breve{\Theta})^{3}$ vector functions. From $[1,6,29]$ we have the following two lemmas. 
Lemma 1 For $\breve{v}(r, \theta, z) \in \breve{H}^{s}(\breve{\Theta}), s=0,1$, the mapping $\breve{v}(r, \theta, z) \rightarrow v(r, z) \in{ }_{1} H^{s}(\Theta)$ is well defined for smooth functions and (up to a factor of $\sqrt{2 \pi}$ ) is an isometry. Hence the lifting $v(r, z) \in$ ${ }_{1} H^{s}(\Theta), v(r, z) \rightarrow \breve{v}(r, \theta, z) \in \breve{H}^{s}(\breve{\Theta})$ is also an isometry (up to a factor of $\sqrt{2 \pi}$ ).

For the case $s=2$ introduce

$$
{ }_{1} H_{+}^{2}(\Theta)=\left\{v \in{ }_{1} H^{2}(\Theta): \partial_{r} v / r \in{ }_{1} L^{2}(\Theta)\right\}
$$

Lemma 2 For $\breve{v}(r, \theta, z) \in \breve{H}^{2}(\breve{\Theta})$, the mapping $\breve{v}(r, \theta, z) \rightarrow v(r, z) \in{ }_{1} H_{+}^{2}(\Theta)$ is (up to a factor of $\sqrt{2 \pi}$ ) an isometry.

The trace space ${ }_{1} H^{1 / 2}(\Gamma)$ is defined using $\breve{H}^{1 / 2}(\breve{\Gamma})$ and the isometry between $\breve{H}^{1}(\breve{\Theta})$ and ${ }_{1} H^{1}(\Theta)$, and ${ }_{1} H^{-1 / 2}(\Gamma)$ the dual space of ${ }_{1} H^{1 / 2}(\Gamma)$.

Let $\nabla_{a}:=[\partial / \partial r, \partial / \partial z]^{T}$, and for $\mathbf{v}=\left(v_{r}, v_{z}\right), \mathbf{d}_{a}(\mathbf{v}):=1 / 2\left(\nabla_{a}(\mathbf{v})+\left(\nabla_{a}(\mathbf{v})\right)^{T}\right)$, and $\operatorname{div}_{a x i}(\mathbf{v}):=$ $\nabla_{a} \cdot \mathbf{v}+v_{r} / r$. In addition,

$$
H\left(d i v_{a x i}, \Theta\right):=\left\{\mathbf{v}=\left(v_{r}, v_{z}\right) \in\left({ }_{1} L^{2}(\Theta)\right)^{2}: \operatorname{div} v_{a x i} \mathbf{v} \in{ }_{1} L^{2}(\Theta)\right\} .
$$

For $\mathbf{v} \in H\left(d i v_{a x i}, \Theta\right),\|\mathbf{v}\|_{H\left(d i v_{a x i}, \Theta\right)}:=\left(\left\|\operatorname{div}_{a x i}(\mathbf{v})\right\|_{1 L^{2}(\Theta)}^{2}+\left\|v_{r}\right\|_{1 L^{2}(\Theta)}^{2}+\left\|v_{z}\right\|_{1 L^{2}(\Theta)}^{2}\right)^{1 / 2}$. Analogous to Lemma 2, we have the following relationship between $H\left(\operatorname{div}_{a x i}, \Theta\right)$ and $\breve{H}(\operatorname{div}, \breve{\Theta}):=\{\breve{\mathbf{v}} \in$ $\left.\breve{L}^{2}(\breve{\Theta})^{3}: \nabla \cdot \breve{\mathbf{v}} \in L^{2}(\breve{\Theta})\right\}$, where $\|\breve{\mathbf{v}}\|_{H(\operatorname{div}, \breve{\Theta})}:=\left(\|\operatorname{div}(\breve{\mathbf{v}})\|_{L^{2}(\breve{\Theta})}^{2}+\left\|v_{x}\right\|_{L^{2}(\breve{\Theta})}^{2}+\left\|v_{y}\right\|_{L^{2}(\breve{\Theta})}^{2}+\left\|v_{z}\right\|_{L^{2}(\breve{\Theta})}^{2}\right)^{1 / 2}$.

Lemma 3 For $\breve{\mathbf{v}}(r, \theta, z)=\left(\breve{v}_{r}, \breve{v}_{\theta}, \breve{v}_{z}\right) \in \breve{H}(\operatorname{div}, \breve{\Theta})$, the mapping $\left(\breve{v}_{r}, 0, \breve{v}_{z}\right) \rightarrow\left(v_{r}, v_{z}\right)=\mathbf{v}(r, z) \in$ $H\left(\right.$ div $\left._{a x i}, \Theta\right)$ is (up to a factor of $\sqrt{2 \pi}$ ) an isometry.

For the description of the fluid flow in $\Omega_{f}$, we introduce the space ${ }_{1} V^{1}(\Theta)$, a subset of ${ }_{1} H^{l}(\Theta)$, given by

${ }_{1} V^{1}(\Theta)=\left\{w \in{ }_{1} H^{1}(\Theta): w \in{ }_{-1} L^{2}(\Theta)\right\}, \quad$ with norm $\|w\|_{1} V^{1}(\Theta)=\left(|w|_{1}^{2} H^{1}(\Theta)+\|w\|_{-1}^{2} L^{2}(\Theta)\right)^{1 / 2}$.

The relevance of the space ${ }_{1} V^{1}(\Theta)$ is apparent from the following lemma $[1,6,29]$.

Lemma 4 For $\breve{\mathbf{v}}(r, \theta, z)=\left(\breve{v}_{r}, \breve{v}_{\theta}, \breve{v}_{z}\right) \in \breve{H}^{1}(\breve{\Theta})^{3}$, we have $\left(\breve{v}_{r}, \breve{v}_{z}\right) \rightarrow\left(v_{r}, v_{z}\right) \in{ }_{1} V^{1}(\Theta) \times{ }_{1} H^{1}(\Theta)$.

In order to incorporate the homogeneous boundary condition for the velocity on $\Gamma_{f}$, let ${ }_{1} H_{\diamond}^{1}\left(\Omega_{f}\right)=\left\{w \in{ }_{1} H^{1}\left(\Omega_{f}\right): w=0\right.$ on $\left.\Gamma_{f}\right\}, \quad$ and ${ }_{1} V_{\diamond}^{1}\left(\Omega_{f}\right)=\left\{w \in{ }_{1} V^{1}\left(\Omega_{f}\right): w=0\right.$ on $\left.\Gamma_{f}\right\}$. For convenience of notation, let $X_{f}:={ }_{1} V_{\diamond}^{1}\left(\Omega_{f}\right) \times_{1} H_{\diamond}^{1}\left(\Omega_{f}\right),\|\mathbf{v}\|_{X_{f}}=\left(\left\|v_{r}\right\|_{1 V^{1}\left(\Omega_{f}\right)}^{2}+\left|v_{z}\right|_{1}^{2} H^{1}\left(\Omega_{f}\right)\right)^{1 / 2}$, and $M_{f}:={ }_{1} L^{2}\left(\Omega_{f}\right)$ with $\|\cdot\|_{M_{f}}=\|\cdot\|_{1} L^{2}\left(\Omega_{f}\right)$.

The underlying space for the fluid velocity in $\Omega_{p}$ is $H\left(\operatorname{div}_{\text {axi }}, \Omega_{p}\right)$. However a function $\mathbf{w}$ in this space may not have sufficient regularity for its trace to exist on $\partial \Omega_{p}$. Hence, the interpretation of 
the boundary condition $\left.\mathbf{w} \cdot \mathbf{n}\right|_{\Gamma_{p}}=0$ needs to be carefully defined. For $\breve{\mathbf{v}} \in \breve{H}\left(\operatorname{div}, \breve{\Omega}_{p}\right)$ we have that $\breve{\mathbf{v}} \cdot \breve{\mathbf{n}} \in \breve{H}^{-1 / 2}\left(\breve{\Omega}_{p}\right)$. For $\mathbf{v} \in H\left(\operatorname{div}_{a x i}, \Omega_{p}\right), \lambda \in{ }_{1} H^{1 / 2}(\Gamma)$, following Galvis and Sarkis [19] (see also [17]), we define the operator $\mathbf{v} \cdot \mathbf{n}_{p} \in{ }_{1} H^{-1 / 2}(\Gamma)$ via an extension operator $E_{\Gamma} \lambda$. Specifically, for $\breve{\lambda} \in \breve{H}^{1 / 2}(\Gamma)$ the axisymmetric lifting of $\lambda$ from $\Gamma$ to $\breve{\Gamma}$, define $\breve{E}_{\breve{\Gamma}} \breve{\lambda}:=\gamma_{0} \breve{\varphi}$, where $\gamma_{0}$ is the trace operator from $\breve{H}^{1}\left(\breve{\Omega}_{p}\right)$ to $\breve{H}^{1 / 2}\left(\partial \breve{\Omega}_{p}\right)$, and $\breve{\varphi} \in \breve{H}^{1}\left(\breve{\Omega}_{p}\right)$ is the weak solution of

$$
\begin{gathered}
-\nabla \cdot \nabla \breve{\varphi}=0, \quad \text { in } \breve{\Omega}_{p}, \\
\breve{\varphi}=\left\{\begin{array}{ll}
\breve{\lambda}, & \text { on } \breve{\Gamma} \\
0, & \text { on } \breve{\Gamma}_{\text {out }}
\end{array}, \quad \partial \breve{\varphi} / \partial \mathbf{n}_{p}=0, \text { on } \partial \breve{\Omega}_{p} \backslash\left(\breve{\Gamma} \cup \breve{\Gamma}_{\text {out }}\right) .\right.
\end{gathered}
$$

$E_{\Gamma} \lambda$ is the axisymmetric restriction of $\breve{E}_{\breve{\Gamma}} \breve{\lambda}$ to $\partial \Omega_{p}$, satisfying $\left\|E_{\Gamma} \lambda\right\|_{1 H^{1 / 2}\left(\partial \Omega_{p}\right)} \leq C\|\lambda\|_{1 H^{1 / 2}(\Gamma)}$. Then, we define the operator $\mathbf{v} \cdot \mathbf{n} \in{ }_{1} H^{-1 / 2}(\Gamma)$ as

$$
\langle\mathbf{v} \cdot \mathbf{n}, \lambda\rangle_{\Gamma}:=\left\langle\mathbf{v} \cdot \mathbf{n}, E_{\Gamma} \lambda\right\rangle_{\partial \Omega_{p}}=\frac{1}{2 \pi}\left\langle\breve{\mathbf{v}} \cdot \breve{\mathbf{n}}_{p}, \breve{E}_{\breve{\Gamma}} \breve{\lambda}\right\rangle_{\partial \breve{\Omega}_{p}}
$$

where $\langle\cdot, \cdot\rangle_{\partial \Omega_{p}}$ denotes the ${ }_{1} L^{2}\left(\partial \Omega_{p}\right)$ inner product, extended to a duality pairing.

For the description of the fluid flow in $\Omega_{p}$, let

$$
\begin{gathered}
X_{p}:=\left\{\mathbf{w} \in H\left(\operatorname{div}_{a x i}, \Omega_{p}\right):\left.\mathbf{w} \cdot \mathbf{n}\right|_{\Gamma_{p}}=0\right\}, \quad M_{p}:={ }_{1} L^{2}\left(\Omega_{p}\right) . \\
\text { and }\|\mathbf{w}\|_{X_{p}}:=\left(\left\|\operatorname{div}_{a x i}(\mathbf{w})\right\|_{1 L^{2}\left(\Omega_{p}\right)}^{2}+\|\mathbf{w}\|_{1 L^{2}\left(\Omega_{p}\right)}^{2}\right)^{1 / 2},\|\cdot\|\left\|_{M_{p}}=\right\| \cdot \|_{1} L^{2}\left(\Omega_{p}\right)
\end{gathered}
$$

Let

$$
X:=X_{f} \times X_{p}, \text { and } \quad M:=\left\{q \in M_{f} \times M_{p}: \int_{\Omega} q r d \mathbf{x}=0\right\},
$$

and denote the dual space of $X$ by $X^{*}$.

The axisymmetric weak formulation for (2.1)-(2.10) may be stated as: Given $\mathbf{f} \in X^{*}, \Xi \in \mathbb{R}$, determine $(\mathbf{u}, p, \lambda, \boldsymbol{\beta}) \in X \times M \times{ }_{1} H^{1 / 2}(\Gamma) \times \mathbb{R}^{2}$ such that, for all $\mathbf{v} \in X$ and $(q, \zeta, \varrho) \in M \times$ ${ }_{1} H^{1 / 2}(\Gamma) \times \mathbb{R}^{2}$,

$$
\begin{aligned}
a(\mathbf{u}, \mathbf{v})-b(\mathbf{v},(p, \boldsymbol{\beta}))+b_{I}(\mathbf{v}, \lambda) & =(\mathbf{f}, \mathbf{v}) \\
b(\mathbf{u},(q, \varrho))-b_{I}(\mathbf{u}, \zeta) & =\varrho \cdot\left[\begin{array}{r}
-1 \\
1
\end{array}\right] \Xi /(2 \pi)
\end{aligned}
$$

where

$$
\begin{aligned}
& a(\mathbf{u}, \mathbf{v}):=a_{f}\left(\mathbf{u}_{f}, \mathbf{v}_{f}\right)+ a_{p}\left(\mathbf{u}_{p}, \mathbf{v}_{p}\right), \quad b(\mathbf{v},(q, \varrho)):=b_{f}\left(\mathbf{v}_{f},\left(q_{f}, \varrho_{1}\right)\right)+b_{p}\left(\mathbf{v}_{p},\left(q_{p}, \varrho_{2}\right)\right) \\
& b_{I}(\mathbf{v}, \zeta):=\int_{\Gamma} \mathbf{v}_{f} \cdot \mathbf{n}_{f} \zeta r d s+\left\langle\mathbf{v}_{p} \cdot \mathbf{n}_{p}, \zeta\right\rangle_{\Gamma}
\end{aligned}
$$


and

$$
\begin{aligned}
a_{f}(\mathbf{u}, \mathbf{v}) & :=\int_{\Omega_{f}} 2 \nu\left(\mathbf{d}_{a}(\mathbf{u}): \mathbf{d}_{a}(\mathbf{v})+\frac{u_{r}}{r} \frac{v_{r}}{r}\right) r d \mathbf{x}+\int_{\Gamma} \alpha_{a s}^{-1}(\mathbf{u} \cdot \mathbf{t})(\mathbf{v} \cdot \mathbf{t}) r d s, \\
a_{p}(\mathbf{u}, \mathbf{v}) & :=\int_{\Omega_{p}} \kappa \mathbf{u} \cdot \mathbf{v} r d \mathbf{x} \\
b_{f}(\mathbf{v},(q, \beta)) & :=\int_{\Omega_{f}} q\left(\nabla_{a} \cdot \mathbf{v}+\frac{v_{r}}{r}\right) r d \mathbf{x}+\beta \int_{\Gamma_{\text {in }}} \mathbf{v} \cdot \mathbf{n}_{f} r d s \\
b_{p}(\mathbf{v},(q, \beta)) & :=\int_{\Omega_{p}} q\left(\nabla_{a} \cdot \mathbf{v}+\frac{v_{r}}{r}\right) r d \mathbf{x}+\beta \int_{\Gamma_{\text {out }}} \mathbf{v} \cdot \mathbf{n}_{p} r d s .
\end{aligned}
$$

In (2.21) $\alpha_{a s}$ is the friction constant from the BJS condition.

Conditions for the existence and uniqueness of a solution to (2.17)-(2.18) are analogous to that for the discrete formulation (3.14)-(3.15). Namely, (i) continuity of the operators $a(\cdot, \cdot), b(\cdot, \cdot)$, and $b_{I}(\cdot, \cdot)$, (ii) the coercivity of $a(\cdot, \cdot)$ (on an appropriate subspace), and (iii) that $b(\cdot, \cdot)$, and $b_{I}(\cdot, \cdot)$ satisfy suitable inf-sup conditions (over appropriate subspaces). The continuity of the operators and the coercivity of $a(\cdot, \cdot)$ are straightforward to show. Below we establish the inf-sup conditions for $b(\cdot, \cdot)$ and $b_{I}(\cdot, \cdot)$ for the discrete approximation problem. Establishing the inf-sup conditions for the continuous setting can be done in a similar manner. (See also $[27,19]$.)

Theorem 1 Given $\mathbf{f} \in X^{*}, \Xi \in \mathbb{R}$, there exists a unique solution $(\mathbf{u}, p, \lambda, \boldsymbol{\beta}) \in X \times M \times{ }_{1} H^{1 / 2}(\Gamma) \times$ $\mathbb{R}^{2}$ satisfying (2.17)-(2.18).

\section{Equivalence of the Differential Equations and Weak Formulation}

The weak formulation (2.17)-(2.24) is obtained by multiplying the differential equations by suitably smooth functions, integrating over the domain, and using Green's theorem. Additionally, integrals over $\breve{\Gamma}_{\text {in }}$ and $\breve{\Gamma}_{\text {out }}$ (arising from using Green's theorem) are replaced by $\beta_{1} \int_{\breve{\Gamma}_{\text {in }}} \breve{\mathbf{v}} \cdot \breve{\mathbf{n}}_{f} d S$ and $\beta_{2} \int_{\breve{\Gamma}_{\text {out }}} \breve{\mathbf{v}}$. $\breve{\mathbf{n}}_{p} d S$, respectively. For a smooth solution the steps used in deriving the weak formulation can be reversed to show that equations (2.1)-(2.4), and (2.5)-(2.8) are satisfied. In addition, a smooth solution to (2.17)-(2.18) satisfies the following boundary conditions (see [18, 17]).

Let $\breve{\mathbf{s}}_{t}$ on $\breve{\Gamma}_{\text {in }}$ be given by

$$
2 \nu \breve{\mathbf{d}}(\breve{\mathbf{u}}) \breve{\mathbf{n}}_{f}=s_{n} \breve{\mathbf{n}}_{f}+\breve{\mathbf{s}}_{t},
$$

where $\breve{s}_{n}:=\left(2 \nu \breve{\mathbf{d}}(\breve{\mathbf{u}}) \breve{\mathbf{n}}_{f}\right) \cdot \breve{\mathbf{n}}_{f}$. Then, smooth solutions to (2.5)-(2.8) satisfy

$$
\begin{array}{ll}
\text { On } \breve{\Gamma}_{\text {in }}: & -\breve{p}_{f}+\breve{s}_{n}=-\beta_{1} \quad \text { and } \quad \breve{\mathbf{s}}_{t}=\mathbf{0} . \\
\text { On } \breve{\Gamma}_{\text {out }}: & \breve{p}_{p}=-\beta_{2} .
\end{array}
$$

\section{Finite Element Approximation}

In this section we discuss the finite element approximation to the coupled axisymmetric StokesDarcy system $(2.17),(2.18)$. We focus our attention on conforming approximating spaces $X_{f, h} \subset X_{f}$, 
$M_{f, h} \subset M_{f}, X_{p, h} \subset X_{p}, M_{p, h} \subset M_{p}, L_{h} \subset{ }_{1} H^{1 / 2}(\Gamma)$, where $X_{f, h}, M_{f, h}$ denote velocity and pressure spaces typically used for fluid flow approximations, and $X_{p, h}, M_{p, h}$ denote velocity and pressure spaces typically used for (mixed formulation) Darcy flow approximations.

We begin by describing the finite element approximation framework used in the analysis.

Let $\Omega_{j} \subset \mathbb{R}^{2}, j=f, p$, be a polygonal domain and let $\mathcal{T}_{j, h}$ be a triangulation of $\overline{\Omega_{j}}$. Thus, the computational domain is defined by

$$
\bar{\Omega}=\cup K ; \quad K \in \mathcal{T}_{f, h} \cup \mathcal{T}_{p, h}
$$

We assume that the triangulation is shape-regular and quasi-uniform, i.e. that there exist constants $c_{1}, c_{2}$ such that

$$
c_{1} h \leq h_{K} \leq c_{2} \rho_{K}
$$

where $h_{K}$ is the diameter of triangle $K, \rho_{K}$ is the diameter of the greatest ball included in $K$, and $h=\max _{K \in \mathcal{T}_{f, h} \cup \mathcal{T}_{p, h}} h_{K}$.

We also assume that the triangulation on $\overline{\Omega_{p}}$ induces the partition on $\Gamma$, which we denote $\mathcal{T}_{\Gamma, h}$.

Let $P_{k}(K)$ denote the space of polynomials on $K$ of degree no greater than $k$, and $R T_{k}(K):=$ $\left(P_{k}(K)\right)^{2}+\mathbf{x} P_{k}(K)$ denote the $k$ th order Raviart-Thomas (R-T) elements [36, 7]. Then we define the finite element spaces as follows.

$$
\begin{aligned}
X_{f, h}:=\left\{\mathbf{v} \in X_{f} \cap C\left(\overline{\Omega_{f}}\right)^{2}:\left.\mathbf{v}\right|_{K} \in P_{m}(K), \forall K \in \mathcal{T}_{f, h}\right\}, \\
M_{f, h}:=\left\{q \in M_{f} \cap C\left(\overline{\Omega_{f}}\right):\left.q\right|_{K} \in P_{m-1}(K), \forall K \in \mathcal{T}_{f, h}\right\}, \\
X_{p, h}:=\left\{\mathbf{v} \in R T_{k}(K), \forall K \in \mathcal{T}_{p, h}\right\} \\
M_{p, h}:=\left\{q \in M_{f}:\left.q\right|_{K} \in P_{k}(K), \forall K \in \mathcal{T}_{p, h}\right\} \\
L_{h}:=\left\{\zeta \in C(\Gamma):\left.\zeta\right|_{K} \in P_{l}(K), \forall K \in \mathcal{T}_{\Gamma, h}\right\} .
\end{aligned}
$$

The spaces $\left(X_{f, h}, M_{f, h}\right)$ represent the Taylor-Hood pair of approximation spaces. The analysis below also holds for $\left(X_{p, h}, M_{p, h}\right)$ corresponding to the Brezzi-Douglas-Marini (BDM) approximating finite element spaces. Analogous to the continuous formulation, we let $X_{h}:=X_{f, h} \times X_{p, h}$, and $M_{h}:=$ $\left\{q \in M_{f, h} \times M_{p, h}: \int_{\Omega} q r d \mathbf{x}=0\right\}$.

Remark: In the axisymmetric setting, for the construction of the R-T interpolant weighted integrals, i.e. $\int_{\partial K} \ldots r d s, \int_{K} \ldots r d \mathbf{x}$, are used. (See [15].)

Below we assume that $m \geq 2, k \geq 1$, and $l \leq k$.

Note that the interfacial pressure approximating space $L_{h}$ is contained in the space of the trace of the normal component of the velocities of $X_{p, h}$, i.e. $L_{h} \subset\left\{\left.\mathbf{v} \cdot \mathbf{n}_{p}\right|_{\Gamma}: \mathbf{v} \in X_{p, h}\right\}$.

Used in the analysis below are the following two interpolation properties.

1. From [4], there exists a generalized Clément interpolation operator $I_{C}:{ }_{1} H^{1}\left(\Omega_{i}\right) \rightarrow\left(\Pi_{K \in \mathcal{T}_{i, h}} P_{s}(K)\right) \cap$ $C\left(\Omega_{i}\right)$ such that

$$
\left\|v-I_{c} v\right\|_{1 L^{2}(\Gamma)} \leq C h^{1 / 2}\|v\|_{1 H^{1}\left(\Omega_{i}\right)}, \quad i \in\{f, p\}, \quad s \geq 1 .
$$

2. From the note above, and the definition of the R-T interpolant, $I_{R T} \mathbf{v}$,

$$
\left\langle\mathbf{v} \cdot \mathbf{n}_{p}, \lambda_{h}\right\rangle_{\Gamma}=\left\langle I_{R T} \mathbf{v} \cdot \mathbf{n}_{p}, \lambda_{h}\right\rangle_{\Gamma}, \quad \text { for all } \lambda_{h} \in L_{h} .
$$


Also used in the analysis are the discrete function space:

$$
\begin{array}{r}
V_{h}:=\left\{\mathbf{v} \in X_{h}: b_{I}\left(\mathbf{v}_{h}, \zeta\right)=0, \text { for all } \zeta \in L_{h}\right\}, \\
Z_{h}:=\left\{\mathbf{v} \in V_{h}: b(\mathbf{v},(q, \varrho))=0, \text { for all }(q, \varrho) \in M_{h} \times \mathbb{R}^{2}\right\}
\end{array}
$$

Let

$$
X_{f, h}^{0}:=\left\{\mathbf{v} \in X_{f, h}:\left.\mathbf{v}\right|_{\partial \Omega_{f} \backslash \Gamma}=\mathbf{0}\right\} .
$$

We have the following lemma.

Lemma 5 There exists $C_{f, h}>0$, such that

$$
\inf _{0 \neq q_{h} \in M_{f, h}} \sup _{\mathbf{v}_{h} \in X_{f, h}^{0}} \frac{\int_{\Omega_{f}} q_{h} d i v_{a x i}\left(\mathbf{v}_{h}\right) r d \mathbf{x}}{\left\|q_{h}\right\|_{M_{f}}\left\|\mathbf{v}_{h}\right\|_{X_{f}}} \geq C_{f, h} .
$$

Proof For the case of the pressure space having mean value equal to zero the inf-sup condition (3.10) is established in [28]. As commented in [27], one can extend the inf-sup condition to the above pressure space via a local projector operator argument. (See [7], Section VI.4.)

For $\left(X_{p, h}, M_{p, h}\right)$ Raviart-Thomas approximation spaces for the velocity and pressure, unlike in the Cartesian setting, $a_{p}(\cdot, \cdot)$ is not coercive, with respect to the $H\left(d i v, \Omega_{p}\right)$ norm, on

$$
Z_{p, h}:=\left\{\mathbf{v} \in X_{p, h}: \int_{\Omega_{p}} q \operatorname{div}_{a x i}(\mathbf{v}) r d \mathbf{x}=0, \forall q \in M_{p, h}\right\}
$$

To compensate for this we add the term

$$
\gamma \int_{\Omega_{p}} \operatorname{div}_{a x i}(\mathbf{u}) d i v_{a x i}(\mathbf{v}) r d \mathbf{x}
$$

to $a_{p}(\mathbf{u}, \mathbf{v})$, where $\gamma>0$ is a fixed constant, and define

$$
a_{p, \gamma}(\mathbf{u}, \mathbf{v}):=a_{p}(\mathbf{u}, \mathbf{v})+\gamma \int_{\Omega_{p}} \operatorname{div}_{a x i}(\mathbf{u}) \operatorname{div} v_{a x i}(\mathbf{v}) r d \mathbf{x}
$$

In the approximation of Stokes and Navier-Stokes fluid flow problems in the Cartesian setting, the addition of the analogous term to (3.12) has received considerable attention recently as a means of improving the pointwise mass conservation of the approximation. (See [33, 34, 32, 26, 10].)

In case an axisymmetric source term, $\breve{g}$, is modeled in the porous domain $\breve{\Omega}_{p}$, i.e. in place of (2.6) we have the equation $\nabla \cdot \breve{\mathbf{u}}_{p}=\breve{g}$ in $\breve{\Omega}_{p}$, together with adding (3.12) to $a_{p}(\mathbf{u}, \mathbf{v})$ the term $\gamma \int_{\Omega_{p}} g d i v_{a x i}(\mathbf{v}) r d \mathbf{x}$ would be added to the right hand side of (3.14).

Discrete Approximation Problem: Given $\mathbf{f} \in X^{*}, \Xi \in \mathbb{R}$, determine $\left(\mathbf{u}_{h}, p_{h}, \lambda_{h}, \boldsymbol{\beta}\right) \in\left(X_{h} \times M_{h} \times\right.$

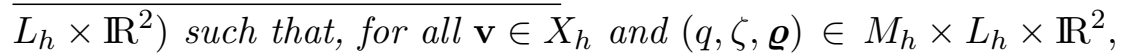

$$
\begin{aligned}
a_{\gamma}\left(\mathbf{u}_{h}, \mathbf{v}\right)-b\left(\mathbf{v},\left(p_{h}, \boldsymbol{\beta}\right)\right)+b_{I}\left(\mathbf{v}, \lambda_{h}\right) & =(\mathbf{f}, \mathbf{v}), \\
b\left(\mathbf{u}_{h},(q, \boldsymbol{\varrho})\right)-b_{I}\left(\mathbf{u}_{h}, \zeta\right) & =\boldsymbol{\varrho} \cdot\left[\begin{array}{r}
-1 \\
1
\end{array}\right] \Xi /(2 \pi),
\end{aligned}
$$


where $a_{\gamma}(\mathbf{u}, \mathbf{v}):=a_{f}\left(\mathbf{u}_{f}, \mathbf{v}_{f}\right)+a_{p, \gamma}\left(\mathbf{u}_{p}, \mathbf{v}_{p}\right)$.

A necessary condition for the existence and uniqueness of solutions to (3.14)-(3.15) is that, for the discrete spaces $X_{h}, M_{h}$, and $L_{h}$, the following two inf-sup conditions are satisfied. There exists constants $C_{b h}, C_{X \Gamma h}>0$.

$$
\begin{aligned}
\inf _{(0, \mathbf{0}) \neq\left(q_{h}, \boldsymbol{\beta}\right) \in M_{h} \times \mathbb{R}^{2} \mathbf{v}_{h} \in V_{h}} \frac{b\left(\mathbf{v}_{h},\left(q_{h}, \boldsymbol{\beta}\right)\right)}{\left\|\mathbf{v}_{h}\right\|_{X}\|(q, \boldsymbol{\beta})\|_{M \times \mathbb{R}^{2}}} \geq C_{b h}, \\
\inf _{\operatorname{ind}_{0 \neq \lambda_{h} \in L_{h}} \sup _{\mathbf{u}_{h} \in X_{h}} \frac{b_{I}\left(\mathbf{u}_{h}, \lambda_{h}\right)}{\left\|\mathbf{u}_{h}\right\|_{X}\left\|\lambda_{h}\right\|_{1} H^{1 / 2}(\Gamma)}} \geq C_{X \Gamma h .} .
\end{aligned}
$$

Note that the inf-sup conditions (3.16)-(3.17) differ from those in the Cartesian formulations in the operators involved, and the functions spaces and norms used.

The following lemma is helpful in establishing (3.16).

Lemma 6 There exists $C_{R X h}>0$ such that for $h$ sufficiently small

$$
\inf _{\mathbf{0} \neq \boldsymbol{\beta} \in \mathbb{R}^{2} \operatorname{wup}_{h} \in V_{h}} \frac{\beta_{1} \int_{\Gamma_{\text {in }}} \mathbf{w}_{f, h} \cdot \mathbf{n}_{f} r d s+\beta_{2} \int_{\Gamma_{\text {out }}} \mathbf{w}_{p, h} \cdot \mathbf{n}_{p} r d s}{\left\|\mathbf{w}_{h}\right\|_{X}\|\boldsymbol{\beta}\|_{\mathbb{R}^{2}}} \geq C_{R X h} .
$$

Proof: Note that the inf-sup condition (3.18) is equivalent to showing that there exists non-zero $\mathbf{v}_{f, h} \in X_{f, h}$ and $\mathbf{v}_{p, h} \in X_{p, h}$, and constants $C_{1}, C_{2}>0$, such that

$$
\begin{aligned}
\mathbf{v}_{f, h} \cdot \mathbf{n}_{f}=0 \quad \text { on } \Gamma, & \int_{\Gamma_{\text {in }}} \mathbf{v}_{f, h} \cdot \mathbf{n}_{f} r d s \geq C_{1}\left\|\mathbf{v}_{f, h}\right\|_{X_{f}}, \\
\text { and } \mathbf{v}_{p, h} \cdot \mathbf{n}_{p}=0 \quad \text { on } \Gamma, \quad & \int_{\Gamma_{\text {out }}} \mathbf{v}_{p, h} \cdot \mathbf{n}_{p} r d s \geq C_{2}\left\|\mathbf{v}_{p, h}\right\|_{X_{p}} .
\end{aligned}
$$

We assume that there is at most one point on $\bar{\Gamma}_{\text {in }}$ and $\bar{\Gamma}_{\text {out }}$ that lies on the symmetry axis $(r=0)$, and that such a point (if it exists) is an endpoint.

For $i \in\{$ in out $\}$, let $s_{i}(\mathbf{x})$ denote an arclength parameter on $\Gamma_{i}$. We have that there exists constants $r_{0, i}, c_{i}$, such that for $\mathbf{x} \in \Gamma_{i}$,

$$
c_{i} s_{i}(\mathbf{x}) \leq r-r_{0, i} \leq s_{i}(\mathbf{x})
$$

Define $\phi_{\text {in }}: \partial \Omega_{f} \rightarrow \mathbb{R}$ by

$$
\phi_{i n}(\mathbf{x})= \begin{cases}\frac{2}{\left|\Gamma_{i n}\right|} s_{i n}(\mathbf{x}), & \mathbf{x} \in \Gamma_{i n}, \quad 0 \leq s_{i n} \leq \frac{\left|\Gamma_{i n}\right|}{2} \\ \frac{2}{\left|\Gamma_{i n}\right|}\left(\left|\Gamma_{i n}\right|-s_{i n}(\mathbf{x})\right), & \mathbf{x} \in \Gamma_{i n}, \quad \frac{\left|\Gamma_{i n}\right|}{2}<s_{i n}(\mathbf{x}) \leq\left|\Gamma_{i n}\right| \\ 0, & \text { otherwise. }\end{cases}
$$

If $\mathbf{n}_{f}$ is discontinuous on $\Gamma_{i n}$, e.g. $\Gamma_{i n}$ has corners, then $\phi_{i n}$ can be constructed on any subset of $\Gamma_{i n}$ on which $\mathbf{n}_{f}$ is continuous.

Let $\mathbf{a} \in{ }_{1} H^{1 / 2}\left(\partial \Omega_{f}\right)$ be given by

$$
\mathbf{a}(\mathbf{x})=\phi_{i n}(\mathbf{x}) \mathbf{n}_{f}
$$


and $\breve{\mathbf{a}}(x, y, z)$ denote the axisymmetric extension of a from $\partial \Omega_{f}$ to $\partial \breve{\Omega}_{f}$. Introduce $\breve{g} \in^{2}\left(\breve{\Omega}_{f}\right)$ as

$$
\breve{g}(x, y, z)=\frac{1}{\left|\breve{\Omega}_{f}\right|^{1 / 2}} \int_{\partial \breve{\Omega}_{f}} \breve{\mathbf{a}} \cdot \breve{\mathbf{n}}_{f} d \breve{s} .
$$

Note that $\|\breve{\mathbf{a}}\|_{H^{1 / 2}\left(\partial \breve{\Omega}_{f}\right)} \leq \pi \operatorname{diam}\left(\breve{\Omega}_{f}\right)\left\|\phi_{i n}\right\|_{H^{1 / 2}\left(\partial \Omega_{f}\right)} \leq C$, and $\|\breve{g}\|_{L^{2}\left(\breve{\Omega}_{f}\right)} \leq \pi \operatorname{diam}\left(\breve{\Omega}_{f}\right) \mid \int_{\partial \Omega_{f}} \mathbf{a}$. $\mathbf{n}_{f} d s \mid \leq C$.

From [19] we have that there exists $\breve{\mathbf{v}}_{f} \in \breve{H}^{1}\left(\breve{\Omega}_{f}\right)^{3}$ such that

$\nabla \cdot \breve{\mathbf{v}}_{f}=\breve{g}$ in $\breve{\Omega}_{f}, \quad \breve{\mathbf{v}}_{f}=\breve{\mathbf{a}}$ on $\partial \breve{\Omega}_{f}, \quad$ and $\left\|\breve{\mathbf{v}}_{f}\right\|_{H^{1}\left(\breve{\Omega}_{f}\right)} \leq C\left(\|\breve{g}\|_{L^{2}\left(\breve{\Omega}_{f}\right)}+\|\breve{\mathbf{a}}\|_{H^{1 / 2}\left(\partial \breve{\Omega}_{f}\right)}\right) \leq C$.

(Remark: The fact that $\breve{\mathbf{v}}_{f} \in \breve{H}^{1}\left(\breve{\Omega}_{f}\right)^{3} \subset H^{1}\left(\breve{\Omega}_{f}\right)^{3}$ follows from the uniqueness of the solution in $H^{1}\left(\breve{\Omega}_{f}\right)^{3}$.)

$\breve{\mathbf{v}}_{p} \in \breve{H}^{1}\left(\breve{\Omega}_{p}\right)^{3}$ is constructed in an analogous manner to $\breve{\mathbf{v}}_{f}$.

Let $\mathbf{v}_{f}(r, z)=\breve{\mathbf{v}}_{f}(x, y, z), \mathbf{v}_{p}(r, z)=\breve{\mathbf{v}}_{p}(x, y, z)$ and $\left.\mathbf{v}_{f, h}(r, z)=I_{C}\left(\mathbf{v}_{f}(r, z)\right), \mathbf{v}_{p, h}(r, z)\right)=$ $I_{R T}\left(\mathbf{v}_{p}(r, z)\right.$.

$$
\text { Now, } \begin{aligned}
\int_{\Gamma_{i n}} \mathbf{v}_{f, h} \cdot \mathbf{n}_{f} r d s & =\int_{\Gamma_{i n}} \mathbf{v}_{f} \cdot \mathbf{n}_{f} r d s-\int_{\Gamma_{i n}}\left(\mathbf{v}_{f}-\mathbf{v}_{f, h}\right) \cdot \mathbf{n}_{f} r d s \\
& \geq C-C h^{1 / 2}\left\|\mathbf{v}_{f}\right\|_{1} H^{1}\left(\Omega_{f}\right) \\
& \geq C \geq C_{1}\left\|\mathbf{v}_{f, h}\right\|_{X_{f}} .
\end{aligned}
$$

Inequality (3.20) is analogously established.

Lemma 7 For $h$ sufficiently small, there exists $C_{b h}>0$ such that

$$
\inf _{(0, \mathbf{0}) \neq\left(q_{h}, \boldsymbol{\beta}\right) \in M_{h} \times \mathbb{R}^{2} \sup _{h} \in V_{h}} \frac{b\left(\mathbf{v}_{h},\left(q_{h}, \boldsymbol{\beta}\right)\right)}{\left\|\mathbf{v}_{h}\right\|_{X}\|(q, \boldsymbol{\beta})\|_{M \times \mathbb{R}^{2}}} \geq C_{b h} .
$$

Proof: Let $\left(p_{h}, \boldsymbol{\beta}\right) \in M_{h} \times \mathbb{R}^{2}$. We establish (3.23) via the following four steps.

Step 1. Construct $\hat{\mathbf{u}}_{h} \in V_{h}$ to take care of $\boldsymbol{\beta}$ (i.e. the flow constraint.) (Use Lemma 6).

$\overline{\text { Step 2. }}$. Construct $\tilde{\mathbf{u}}_{f, h} \in X_{h}^{0}$ such that $b_{f}\left(\tilde{\mathbf{u}}_{f, h},\left(p_{f, h}, \mathbf{0}\right)\right)=\int_{\Omega_{f}} p_{f, h}\left(p_{f, h}-\operatorname{div}_{a x i}\left(\hat{\mathbf{u}}_{f, h}\right)\right) r d \mathbf{x}$.

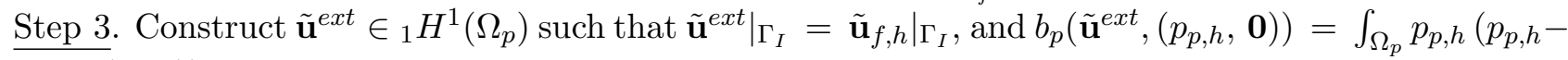
$\left.\operatorname{div}_{a x i}\left(\hat{\mathbf{u}}_{p, h}\right)\right) r d \mathbf{x}$.

Step 4. Let $\tilde{\mathbf{u}}_{p, h}:=I_{R T} \tilde{\mathbf{u}}^{e x t}, \mathbf{u}_{h}=\left(\tilde{\mathbf{u}}_{f, h}, \tilde{\mathbf{u}}_{p, h}\right)+\hat{\mathbf{u}}_{h}$, and verify (3.23) is satisfied.

Step 1. From Lemma 6, there exists $\hat{\mathbf{u}}_{h} \in V_{h}$ such that

$$
\left\|\hat{\mathbf{u}}_{h}\right\|_{X} \leq C\|\boldsymbol{\beta}\|_{\mathbb{R}^{2}} \quad \text { and } \int_{\Gamma_{\text {in }}} \beta_{1} \hat{\mathbf{u}}_{f, h} \cdot \mathbf{n}_{f} r d s+\int_{\Gamma_{\text {out }}} \beta_{2} \hat{\mathbf{u}}_{p, h} \cdot \mathbf{n}_{p} r d s \geq C_{R X h}\|\boldsymbol{\beta}\|_{\mathbb{R}^{2}}^{2}
$$

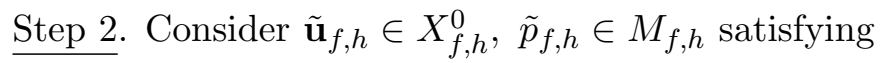

$$
\begin{aligned}
\tilde{a}_{f}\left(\tilde{\mathbf{u}}_{f, h}, \mathbf{v}\right) & -b_{f}\left(\mathbf{v},\left(\tilde{p}_{f, h}, 0\right)\right)=0, \quad \forall \mathbf{v} \in X_{f, h}^{0}, \\
b_{f}\left(\tilde{\mathbf{u}}_{f, h},(q, 0)\right) & =\left(q, p_{f, h}-\operatorname{div}_{a}\left(\hat{\mathbf{u}}_{f, h}\right)\right), \quad \forall q \in M_{f, h},
\end{aligned}
$$


where $\tilde{a}_{f}(\mathbf{u}, \mathbf{v}):=\int_{\Omega_{f}}\left(\nabla_{a} \mathbf{u}: \nabla_{a} \mathbf{v}+\frac{u_{r}}{r} \frac{v_{r}}{r}\right) r d \mathbf{x}$. The existence and uniqueness of $\tilde{\mathbf{u}}_{f, h}$ and $\tilde{p}_{f, h} \in M_{f, h}$ follows from the inf-sup condition (3.10) and the coercivity of $\tilde{a}_{f}(\cdot, \cdot)$. Next, note that

$$
\begin{aligned}
c\left\|\tilde{\mathbf{u}}_{f, h}\right\|_{X_{f}}^{2} & \leq \tilde{a}_{f}\left(\tilde{\mathbf{u}}_{f, h}, \tilde{\mathbf{u}}_{f, h}\right)=b_{f}\left(\tilde{\mathbf{u}}_{f, h},\left(\tilde{p}_{f, h}, 0\right)\right) \\
& =\int_{\Omega_{f}} \tilde{p}_{f, h}\left(p_{f, h}-\operatorname{div}_{a x i}\left(\hat{\mathbf{u}}_{f, h}\right)\right) r d \mathbf{x} \\
& \leq\left\|\tilde{p}_{f, h}\right\|_{M_{f}}\left(\left\|p_{f, h}\right\|_{M_{f}}+\left\|\operatorname{div}_{a x i}\left(\hat{\mathbf{u}}_{f, h}\right)\right\|_{1 L^{2}\left(\Omega_{f}\right)}\right) \\
& \leq\left\|\tilde{p}_{f, h}\right\|_{M_{f}}\left(\left\|p_{f, h}\right\|_{M_{f}}+C\left\|\hat{\mathbf{u}}_{f, h}\right\|_{X_{f}}\right) \\
& \leq C\left\|\tilde{p}_{f, h}\right\|_{M_{f}}\left(\left\|p_{f, h}\right\|_{M_{f}}+\|\boldsymbol{\beta}\|_{\mathbb{R}^{2}}\right) .
\end{aligned}
$$

From the inf-sup condition (3.10),

$$
\begin{aligned}
C_{f, h}\left\|\tilde{p}_{f, h}\right\|_{M_{f}} & \leq \sup _{\mathbf{v} \in X_{f, h}^{0}} \frac{\int_{\Omega_{f}} \tilde{p}_{f, h} \operatorname{div} v_{a x i}(\mathbf{v}) r d \mathbf{x}}{\|\mathbf{v}\|_{X_{f}}}=\sup _{\mathbf{v} \in X_{f, h}^{0}} \frac{\int_{\Omega_{f}}\left(\nabla_{a} \tilde{\mathbf{u}}_{f, h}: \nabla_{a} \mathbf{v}+\frac{\tilde{u}_{f, h, r}}{r} \frac{v_{r}}{r}\right) r d \mathbf{x}}{\|\mathbf{v}\|_{X_{f}}} \\
& \leq \sup _{\mathbf{v} \in X_{f, h}^{0}} \frac{\left\|\tilde{\mathbf{u}}_{f, h}\right\|_{X_{f}}\|\mathbf{v}\|_{X_{f}}}{\|\mathbf{v}\|_{X_{f}}}=\left\|\tilde{\mathbf{u}}_{f, h}\right\|_{X_{f}} .
\end{aligned}
$$

Combining (3.27) and (3.28) we obtain

$$
\left\|\tilde{\mathbf{u}}_{f, h}\right\|_{X_{f}} \leq C\left(\left\|p_{f, h}\right\|_{M_{f}}+\|\boldsymbol{\beta}\|_{\mathbb{R}^{2}}\right) .
$$

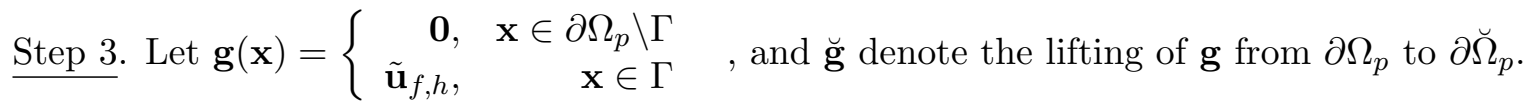

As, for $\mathbf{x} \in \Gamma, \lim _{\mathbf{x} \rightarrow \bar{\Gamma} \cap \partial \Omega_{p}} \tilde{\mathbf{u}}_{f, h}=\mathbf{0}$, then $\breve{\mathbf{g}} \in \breve{H}^{1 / 2}\left(\partial \breve{\Omega}_{p}\right)^{3}$. Also,

$$
\begin{aligned}
\|\breve{\mathbf{g}}\|_{\breve{H}^{1 / 2}\left(\partial \breve{\Omega}_{p}\right)^{3}} & \leq C\left\|\breve{\mathbf{u}}_{f, h}\right\|_{H^{1 / 2}(\breve{\Gamma})} \leq C\left\|\breve{\mathbf{u}}_{f, h}\right\|_{H^{1}\left(\breve{\Omega}_{f}\right)} \\
& \leq C\left\|\tilde{\mathbf{u}}_{f, h}\right\|_{X_{f}} \leq C\left(\left\|p_{f, h}\right\|_{M_{f}}+\|\boldsymbol{\beta}\|_{\mathbb{R}^{2}}\right)
\end{aligned}
$$

Let $\breve{\mathbf{z}} \in \breve{H}^{1}\left(\breve{\Omega}_{p}\right)^{3}$ denote an extension of $\breve{\mathbf{g}}$ such that

$$
\left.\breve{\mathbf{z}}\right|_{\partial \breve{\Omega}_{p}}=\left.\breve{\mathbf{g}}\right|_{\partial \breve{\Omega}_{p}} \text {, and }\|\breve{\mathbf{z}}\|_{H^{1}\left(\breve{\Omega}_{p}\right)} \leq C\|\breve{\mathbf{g}}\|_{H^{1 / 2}\left(\partial \breve{\Omega}_{p}\right)}
$$

Let $\breve{\mathbf{w}} \in \breve{H}_{0}^{1}\left(\breve{\Omega}_{p}\right)^{3}, \breve{t} \in_{0}^{2}\left(\breve{\Omega}_{p}\right)$ satisfy

$$
\begin{aligned}
& \int_{\breve{\Omega}_{p}} \nabla \breve{\mathbf{w}}: \nabla \breve{\mathbf{v}} d \breve{\Omega}_{p}-\int_{\breve{\Omega}_{p}} \breve{t} \nabla \cdot \breve{\mathbf{v}} d \breve{\Omega}_{p}=0, \quad \forall \breve{\mathbf{v}} \in H_{0}^{1}\left(\breve{\Omega}_{p}\right)^{3} \\
& \int_{\breve{\Omega}_{p}} \breve{q} \nabla \cdot \breve{\mathbf{w}} d \breve{\Omega}_{p}=\int_{\breve{\Omega}_{p}} \breve{q}\left(\breve{p}_{p, h}-\nabla \cdot \breve{\hat{\mathbf{u}}}_{h}-\nabla \cdot \breve{\mathbf{z}}\right) d \breve{\Omega}_{p}, \quad \forall \breve{q} \in L_{0}^{2}\left(\breve{\Omega}_{p} \gamma\right.
\end{aligned}
$$

(Remark: The fact that $(\breve{\mathbf{w}}, \breve{t}) \in\left(\breve{H}_{0}^{1}\left(\breve{\Omega}_{p}\right)^{3} \times{ }_{0}^{2}\left(\breve{\Omega}_{p}\right)\right) \subset\left(H_{0}^{1}\left(\breve{\Omega}_{p}\right)^{3} \times L_{0}^{2}\left(\breve{\Omega}_{p}\right)\right.$, follows from the uniqueness of the solution of (3.32), and (3.33) in $\left(H_{0}^{1}\left(\Omega_{p}\right)^{3} \times L_{0}^{2}\left(\Omega_{p}\right)\right.$.) 
From the Brezzi theory for mixed variational problems [7], we have the bound

$$
\begin{aligned}
\|\nabla \breve{\mathbf{w}}\|_{L^{2}\left(\breve{\Omega}_{p}\right)} & \leq C\left(\left\|\breve{p}_{p, h}\right\|_{L^{2}\left(\breve{\Omega}_{p}\right)}+\left\|\nabla \cdot \breve{\hat{\mathbf{u}}}_{h}\right\|_{L^{2}\left(\breve{\Omega}_{p}\right)}+\|\nabla \cdot \breve{\mathbf{z}}\|_{L^{2}\left(\breve{\Omega}_{p}\right)}\right) \\
& \leq C\left(\left\|p_{p, h}\right\|_{M_{p}}+\|\boldsymbol{\beta}\|_{\mathbb{R}^{2}}+\left\|p_{f, h}\right\|_{M_{f}}\right) .
\end{aligned}
$$

Let $\tilde{\mathbf{u}}^{\text {ext }}$ denote the reduction of $(\breve{\mathbf{w}}+\breve{\mathbf{z}})$ from $\breve{\Omega}_{p}$ to $\Omega_{p}$. From $(3.30),(3.31)$ and $(3.34)$, $\tilde{\mathbf{u}}^{\text {ext }} \in$

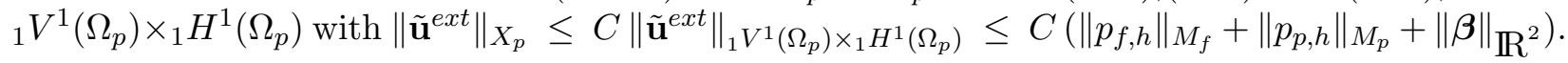

Step 4. Let $\tilde{\mathbf{u}}_{p, h}:=I_{R T} \tilde{\mathbf{u}}^{e x t}, \tilde{\mathbf{u}}_{h}=\left(\tilde{\mathbf{u}}_{f, h}, \tilde{\mathbf{u}}_{p, h}\right)$ and $\mathbf{u}_{h}=\tilde{\mathbf{u}}_{h}+\hat{\mathbf{u}}_{h}$. Next we show that $\tilde{\mathbf{u}}_{h} \in V_{h}$, $\overline{\text { i.e. } b_{I}}\left(\tilde{\mathbf{u}}_{h}, \lambda_{h}\right)=0$, and hence that $\mathbf{u}_{h} \in V_{h}$.

$$
\begin{aligned}
b_{I}\left(\tilde{\mathbf{u}}_{h}, \lambda_{h}\right) & =\int_{\Gamma} \tilde{\mathbf{u}}_{f, h} \cdot \mathbf{n}_{f} \lambda_{h} r d s+\left\langle\tilde{\mathbf{u}}_{p, h} \cdot \mathbf{n}_{p}, E_{\Gamma} \lambda_{h}\right\rangle_{\partial \Omega_{p}} \\
& =\int_{\Gamma} \tilde{\mathbf{u}}_{f, h} \cdot \mathbf{n}_{f} \lambda_{h} r d s+\int_{\Gamma} I_{R T} \tilde{\mathbf{u}}^{e x t} \cdot \mathbf{n}_{p} \lambda_{h} r d s \quad\left(\operatorname{as} I_{R T} \tilde{\mathbf{u}}^{e x t} \cdot \mathbf{n}_{p}=0 \text { on } \partial \Omega_{p} \backslash \Gamma\right) \\
& =\int_{\Gamma} \tilde{\mathbf{u}}_{f, h} \cdot \mathbf{n}_{f} \lambda_{h} r d s+\int_{\Gamma}(\mathbf{w}+\mathbf{z}) \cdot \mathbf{n}_{p} \lambda_{h} r d s \\
& =\int_{\Gamma} \tilde{\mathbf{u}}_{f, h} \cdot \mathbf{n}_{f} \lambda_{h} r d s+\int_{\Gamma} \tilde{\mathbf{u}}_{f, h} \cdot \mathbf{n}_{p} \lambda_{h} r d s=0 .
\end{aligned}
$$

Now,

$$
\begin{aligned}
& \sup _{\mathbf{v}_{h} \in X_{h}} \frac{b\left(\mathbf{v}_{h},\left(p_{h}, \boldsymbol{\beta}\right)\right)}{\left\|\mathbf{v}_{h}\right\|_{X}} \geq \frac{b\left(\mathbf{u}_{h},\left(p_{h}, \boldsymbol{\beta}\right)\right)}{\left\|\mathbf{u}_{h}\right\|_{X}}=\frac{b_{f}\left(\left(\tilde{\mathbf{u}}_{f, h}+\hat{\mathbf{u}}_{f, h}\right),\left(p_{f, h}, \beta_{1}\right)\right)+b_{p}\left(\left(\tilde{\mathbf{u}}_{p, h}+\hat{\mathbf{u}}_{p, h}\right),\left(p_{p, h}, \beta_{2}\right)\right)}{\left\|\mathbf{u}_{h}\right\|_{X}} . \\
& b_{f}\left(\left(\tilde{\mathbf{u}}_{f, h}+\hat{\mathbf{u}}_{f, h}\right),\left(p_{f, h}, \beta_{1}\right)\right)=\int_{\Omega_{f}} p_{f, h} \operatorname{div}_{a x i}\left(\tilde{\mathbf{u}}_{f, h}+\hat{\mathbf{u}}_{f, h}\right) r d \mathbf{x}+\beta_{1} \int_{\Gamma_{i n}}\left(\tilde{\mathbf{u}}_{f, h}+\hat{\mathbf{u}}_{f, h}\right) \cdot \mathbf{n}_{f} r d s \\
& =\int_{\Omega_{f}} p_{f, h}^{2} r d \mathbf{x}+\beta_{1} \int_{\Gamma_{i n}} \hat{\mathbf{u}}_{f, h} \cdot \mathbf{n}_{f} r d s . \\
& b_{p}\left(\left(\tilde{\mathbf{u}}_{p, h}+\hat{\mathbf{u}}_{p, h}\right),\left(p_{p, h}, \beta_{2}\right)\right)=\int_{\Omega_{p}} p_{p, h} \operatorname{div} v_{a x i}\left(\tilde{\mathbf{u}}_{p, h}+\hat{\mathbf{u}}_{p, h}\right) r d \mathbf{x}+\beta_{2} \int_{\Gamma_{\text {out }}}\left(\tilde{\mathbf{u}}_{p, h}+\hat{\mathbf{u}}_{p, h}\right) \cdot \mathbf{n}_{p} r d s \\
& =\int_{\Omega_{p}} p_{p, h} \operatorname{div}_{a x i}\left(I_{R T}(\mathbf{w}+\mathbf{z})\right) r d \mathbf{x}+\int_{\Omega_{p}} p_{p, h} \operatorname{div}_{a x i}\left(\hat{\mathbf{u}}_{p, h}\right) r d \mathbf{x}+\beta_{2} \int_{\Gamma_{\text {out }}} \hat{\mathbf{u}}_{p, h} \cdot \mathbf{n}_{p} r d s \\
& =\int_{\Omega_{p}} p_{p, h} \operatorname{div}_{a x i}(\mathbf{w}+\mathbf{z}) r d \mathbf{x}+\int_{\Omega_{p}} p_{p, h} \operatorname{div}_{a x i}\left(\hat{\mathbf{u}}_{p, h}\right) r d \mathbf{x}+\beta_{2} \int_{\Gamma_{\text {out }}} \hat{\mathbf{u}}_{p, h} \cdot \mathbf{n}_{p} r d s \\
& =\int_{\Omega_{p}} p_{p, h}^{2} r d \mathbf{x}+\beta_{2} \int_{\Gamma_{\text {out }}} \hat{\mathbf{u}}_{p, h} \cdot \mathbf{n}_{p} r d s .
\end{aligned}
$$

Thus

$$
\begin{aligned}
b\left(\mathbf{u}_{h},\left(p_{h}, \boldsymbol{\beta}\right)\right) & =\left\|p_{h}\right\|_{M}^{2}+\beta_{1} \int_{\Gamma_{\text {in }}} \hat{\mathbf{u}}_{f, h} \cdot \mathbf{n}_{f} r d s+\beta_{2} \int_{\Gamma_{\text {out }}} \hat{\mathbf{u}}_{p, h} \cdot \mathbf{n}_{p} r d s \\
& \geq\left\|p_{h}\right\|_{M}^{2}+C_{R X h}\|\boldsymbol{\beta}\|_{\mathbb{R}^{2}}^{2} .
\end{aligned}
$$

As $\left\|\mathbf{u}_{h}\right\|_{X} \leq C\left(\left\|p_{h}\right\|_{M}+\|\boldsymbol{\beta}\|_{\mathbb{R}^{2}}\right.$, the stated result follows. 
Lemma 8 There exists $C_{X Г h}>0$ such that for $h$ sufficiently small

$$
\inf _{0 \neq \lambda_{h} \in L_{h}} \sup _{\mathbf{u}_{h} \in X_{h}} \frac{b_{I}\left(\mathbf{u}_{h}, \lambda_{h}\right)}{\left\|\mathbf{u}_{h}\right\|_{X}\left\|\lambda_{h}\right\|_{1 H^{1 / 2}(\Gamma)}} \geq C_{X \Gamma h} .
$$

\section{Proof:}

The analogous continuous inf-sup condition is established by, given $\lambda \in{ }_{1} H^{1 / 2}(\Gamma)$, constructing a suitable $\mathbf{v}=\left(\mathbf{0}, \mathbf{v}_{p}\right) \in X_{f} \times X_{p}$ such that $b_{I}(\mathbf{v}, \lambda) \geq C\|\mathbf{v}\|_{X}\|\lambda\|_{1 H^{1 / 2}(\Gamma)}$. As, for $\lambda_{h} \in L_{h}$, and the Raviart-Thomas interpolant $I_{R T} \mathbf{v}_{p}$, we have $\left\langle\mathbf{v}_{p} \cdot \mathbf{n}_{p}, \lambda_{h}\right\rangle_{\Gamma}=\left\langle I_{R T} \mathbf{v}_{p} \cdot \mathbf{n}_{p}, \lambda_{h}\right\rangle_{\Gamma}$, then for $\mathbf{v}_{h}=\left(\mathbf{0}, I_{R T} \mathbf{v}_{p}\right),(3.39)$ follows.

With the inf-sup conditions given in (3.16) and (3.17) now established, we have the following.

Theorem 2 Given $\mathbf{f} \in X^{*}, f r \in \mathbb{R}$, for $\gamma>0$ there exists a unique solution $\left(\mathbf{u}_{h}, p_{h}, \lambda_{h}, \boldsymbol{\beta}\right) \in$ $\left(X_{h} \times M_{h} \times L_{h} \times \mathbb{R}^{2}\right)$ satisfying (3.14)-(3.15).

\section{Proof:}

The proof follows from the continuity of the operators $a_{\gamma}(\cdot, \cdot), b(\cdot, \cdot, \cdot)$, and $b_{I}(\cdot, \cdot)$, the coercivity of $a_{\gamma}(\cdot, \cdot)$ on $Z_{h} \times Z_{h}$, and the inf-sup conditions (3.16) and (3.17).

\subsection{A Priori Error Estimate}

In this section we derive the a priori error estimate for the approximation $\left(\mathbf{u}_{h}, p_{h}\right)$. Used in establishing the estimate is the following lemma.

Lemma 9 There exists a constant $C_{c}>0$ such that

$$
\inf _{(0,0, \mathbf{0}) \neq\left(q_{h}, \zeta_{h}, \boldsymbol{\varrho}_{h}\right) \in M_{h} \times L_{h} \times \mathbb{R}^{2} \mathbf{v}_{h} \in X_{h}} \frac{b\left(\mathbf{v}_{h}, q_{h}, \varrho_{h}\right)-b_{I}\left(\mathbf{v}_{h}, \zeta_{h}\right)}{\left(\left\|q_{h}\right\|_{M}+\left\|\zeta_{h}\right\|_{1 H^{1 / 2}(\Gamma)}+\left\|\varrho_{h}\right\|_{\mathbb{R}^{2}}\right)\left\|\mathbf{v}_{h}\right\|_{X}} \geq C_{c} .
$$

Proof: The "combined" inf-sup condition (3.40) follows from the individual inf-sup conditions (3.23) and (3.39). (See [17], appendix.)

Theorem 3 For $(\mathbf{u}, p, \lambda, \boldsymbol{\beta})$ satisfying (2.17)-(2.18) and $\left(\mathbf{u}_{h}, p_{h}, \lambda_{h}, \boldsymbol{\beta}_{h}\right)$ satisfying (3.14)-(3.15), and $h$ sufficiently small, there exists a constant $C>0$ such that

$$
\begin{aligned}
\left\|\mathbf{u}-\mathbf{u}_{h}\right\|_{X} & +\left\|p-p_{h}\right\|_{M}+\left\|\beta-\beta_{h}\right\|_{\mathbb{R}^{2}}+\left\|\lambda-\lambda_{h}\right\|_{1 H^{1 / 2}(\Gamma)} \\
& \leq C\left(i n f_{\mathbf{v}_{h} \in X_{h}}\left\|\mathbf{u}-\mathbf{v}_{h}\right\|_{X}+i n f_{q_{h} \in M_{h}}\left\|p-q_{h}\right\|_{M}+i n f_{\zeta_{h} \in L_{h}}\left\|\lambda-\zeta_{h}\right\|_{1 H^{1 / 2}(\Gamma)}()\right.
\end{aligned}
$$

Proof: Subtracting (3.14) from (2.17), and using $\gamma \int_{\Omega_{p}} q \operatorname{div}_{a x i}\left(\mathbf{u}_{p}\right) r d \mathbf{x}=0$, for all $q \in M_{p}$, we have that for $\left(\mathbf{v}_{h}, q_{h}, \mu_{h}\right) \in Z_{h} \times M_{h} \times L_{h}$

$$
\begin{aligned}
a_{\gamma}\left(\mathbf{u}-\mathbf{u}_{h}, \mathbf{v}_{h}\right) & -b\left(\mathbf{v}_{h}, p, \boldsymbol{\beta}\right)+b_{I}\left(\mathbf{v}_{h}, \lambda\right)=0, \\
\Rightarrow \quad a_{\gamma}\left(\mathbf{u}-\mathbf{u}_{h}, \mathbf{v}_{h}\right) & =b\left(\mathbf{v}_{h}, p-q_{h}, \mathbf{0}\right)-b_{I}\left(\mathbf{v}_{h}, \lambda-\mu_{h}\right) .
\end{aligned}
$$


Writing $\left(\mathbf{u}-\mathbf{u}_{h}\right)=\mathbf{e}_{h}=\left(\mathbf{u}-\mathbf{U}_{h}\right)+\left(\mathbf{U}_{h}-\mathbf{u}_{h}\right):=\boldsymbol{\xi}_{h}+\mathbf{E}_{h}, \mathbf{U}_{h} \in Z_{h}$, and with the choice $\mathbf{v}_{h}=\mathbf{E}_{h},(3.42)$ becomes

$$
a_{\gamma}\left(\mathbf{E}_{h}, \mathbf{E}_{h}\right)=-a_{\gamma}\left(\boldsymbol{\xi}_{h}, \mathbf{E}_{h}\right)+b\left(\mathbf{E}_{h}, p-q_{h}, \mathbf{0}\right)-b_{I}\left(\mathbf{E}_{h}, \lambda-\mu_{h}\right) .
$$

For each of the terms in (3.43) we have the following bounds.

$$
\begin{aligned}
& a_{\gamma}\left(\mathbf{E}_{h}, \mathbf{E}_{h}\right) \geq \nu\left\|\mathbf{E}_{f, h}\right\|_{X_{f}}^{2}+\int_{\Gamma} \alpha_{a s}^{-1}\left(\mathbf{E}_{f, h} \cdot \mathbf{t}\right)^{2} r d s+\min \{\kappa, \gamma\}\left\|\mathbf{E}_{p, h}\right\|_{X_{p}}^{2} . \\
& a_{\gamma}\left(\boldsymbol{\xi}_{h}, \mathbf{E}_{h}\right) \leq \nu\left\|\boldsymbol{\xi}_{f, h}\right\|_{X_{f}}^{2}+\frac{\nu}{4}\left\|\mathbf{E}_{f, h}\right\|_{X_{f}}^{2}+\frac{1}{2} \int_{\Gamma} \alpha_{a s}^{-1}\left(\boldsymbol{\xi}_{f, h} \cdot \mathbf{t}\right)^{2} r d s+\frac{1}{2} \int_{\Gamma} \alpha_{a s}^{-1}\left(\mathbf{E}_{f, h} \cdot \mathbf{t}\right)^{2} r d s \\
& +\frac{(\max \{\kappa, \gamma\})^{2}}{2 \min \{\kappa, \gamma\}}\left\|\boldsymbol{\xi}_{p, h}\right\|_{X_{p}}^{2}+\frac{\min \{\kappa, \gamma\}}{2}\left\|\boldsymbol{\xi}_{p, h}\right\|_{X_{p}}^{2} \\
& \leq \frac{\nu}{4}\left\|\mathbf{E}_{f, h}\right\|_{X_{f}}^{2}+\frac{1}{2} \int_{\Gamma} \alpha_{a s}^{-1}\left(\mathbf{E}_{f, h} \cdot \mathbf{t}\right)^{2} r d s+\frac{\min \{\kappa, \gamma\}}{4}\left\|\mathbf{E}_{p, h}\right\|_{X_{p}}^{2} \\
& +C\left(\left\|\boldsymbol{\xi}_{f, h}\right\|_{X_{f}}^{2}+\left\|\boldsymbol{\xi}_{p, h}\right\|_{X_{p}}^{2}\right) \text {. } \\
& b\left(\mathbf{E}_{h}, p-q_{h}, \mathbf{0}\right) \leq\left\|p_{f}-q_{f, h}\right\|_{M_{f}}\left\|\mathbf{E}_{f, h}\right\|_{X_{f}}+\left\|p_{p}-q_{p, h}\right\|_{M_{p}}\left\|\mathbf{E}_{p, h}\right\|_{X_{p}}
\end{aligned}
$$

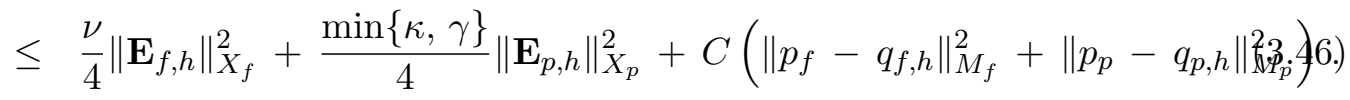

$$
\begin{aligned}
& b_{I}\left(\mathbf{E}_{h}, \lambda-\mu_{h}\right)=\int_{\Gamma}\left(\mathbf{E}_{f, h} \cdot \mathbf{n}_{f}\right)\left(\lambda-\mu_{h}\right) r d s+\left\langle\mathbf{E}_{p, h} \cdot \mathbf{n}_{p},\left(\lambda-\mu_{h}\right)\right\rangle_{\Gamma} \\
& \leq\left\|\mathbf{E}_{f, h} \cdot \mathbf{n}_{f}\right\|_{1 H^{1 / 2}(\Gamma)}\left\|\lambda-\mu_{h}\right\|_{1^{1 / 2}(\Gamma)}+\left\langle\mathbf{E}_{p, h} \cdot \mathbf{n}_{p}, E_{\Gamma}\left(\lambda-\mu_{h}\right)\right\rangle_{\partial \Omega_{p}} \\
& \leq\left\|\mathbf{E}_{f, h}\right\|_{1 H^{1 / 2}(\Gamma)}\left\|\lambda-\mu_{h}\right\|_{1 H^{1 / 2}(\Gamma)}+\left\|\mathbf{E}_{p, h} \cdot \mathbf{n}_{p}\right\|_{1 H^{-1 / 2}\left(\partial \Omega_{p}\right)}\left\|E_{\Gamma}\left(\lambda-\mu_{h}\right)\right\|_{1 H^{1 / 2}\left(\partial \Omega_{p}\right)} \\
& \leq \frac{\nu}{4}\left\|\mathbf{E}_{f, h}\right\|_{X_{f}}^{2}+\frac{\min \{\kappa, \gamma\}}{4}\left\|\mathbf{E}_{p, h}\right\|_{X_{p}}^{2}+C\left\|\lambda-\mu_{h}\right\|_{1 H^{1 / 2}(\Gamma)}^{2} .
\end{aligned}
$$

Combining (3.43)-(3.47),

$$
\begin{aligned}
\left\|\mathbf{E}_{f, h}\right\|_{X_{f}}^{2} & +\left\|\mathbf{E}_{p, h}\right\|_{X_{p}}^{2}+\int_{\Gamma} \alpha_{a s}^{-1}\left(\mathbf{E}_{f, h} \cdot \mathbf{t}\right)^{2} r d s \\
\leq & \left.C\left(\left\|\boldsymbol{\xi}_{f, h}\right\|_{X_{f}}^{2}+\left\|\boldsymbol{\xi}_{p, h}\right\|_{X_{p}}^{2}+\left\|p_{f}-q_{f, h}\right\|_{M_{f}}^{2}+\left\|p_{p}-q_{p, h}\right\|_{M_{p}}^{2}+\left\|\lambda-\mu_{h}\right\|_{1^{1 / 2}(\mathrm{~F})}^{2}\right) 48\right)
\end{aligned}
$$

Using the triangle inequality we obtain

$$
\begin{aligned}
\left\|\mathbf{u}_{f}-\mathbf{u}_{f, h}\right\|_{X_{f}}^{2}+\left\|\mathbf{u}_{p}-\mathbf{u}_{p, h}\right\|_{X_{p}}^{2} \leq 2\left\|\boldsymbol{\xi}_{f, h}\right\|_{X_{f}}^{2}+2\left\|E_{f, h}\right\|_{X_{f}}^{2}+2\left\|\boldsymbol{\xi}_{p, h}\right\|_{X_{p}}^{2}+2\left\|E_{p, h}\right\|_{X_{p}}^{2} \\
\leq C\left(\left\|\boldsymbol{\xi}_{f, h}\right\|_{X_{f}}^{2}+\left\|\boldsymbol{\xi}_{p, h}\right\|_{X_{p}}^{2}+\left\|p_{f}-q_{f, h}\right\|_{M_{f}}^{2}+\left\|p_{p}-q_{p, h}\right\|_{M_{p}}^{2}+\left\|\lambda-\mu_{h}\right\|_{\left.\left.1 H^{1 / 2}\left(\mathbf{(}^{2}\right)\right)^{4}\right)}^{2}\right)
\end{aligned}
$$

As $U_{h} \in Z_{h}, q_{h} \in M_{h}, \mu_{h} \in L_{h}$ are arbitrary, (3.49) implies

$$
\begin{aligned}
\left\|\mathbf{u}_{f}-\mathbf{u}_{f, h}\right\|_{X_{f}}^{2} & +\left\|\mathbf{u}_{p}-\mathbf{u}_{p, h}\right\|_{X_{p}}^{2} \leq C\left(\inf _{\mathbf{v}_{h} \in Z_{h}}\left(\left\|\mathbf{u}_{f}-\mathbf{v}_{f, h}\right\|_{X_{f}}^{2}+\left\|\mathbf{u}_{p}-\mathbf{v}_{p, h}\right\|_{X_{p}}^{2}\right)\right. \\
& \left.+\inf _{q_{h} \in M_{h}}\left(\left\|p_{f}-q_{f, h}\right\|_{M_{f}}^{2}+\left\|p_{p}-q_{p, h}\right\|_{M_{p}}^{2}\right)+\inf _{\mu_{h} \in L_{h}}\left\|\lambda-\mu_{h}\right\|_{1 H^{1 / 2}(\Gamma)}^{2}\right)(3.5
\end{aligned}
$$


The inf-sup condition (3.40) then allows the estimate (3.50) for $\mathbf{v}_{h} \in Z_{h}$ to be lifted to $\mathbf{v}_{h} \in X_{h}$, giving the estimate (3.41) for $\left\|\mathbf{u}-\mathbf{u}_{h}\right\|_{X}$.

To obtain the error estimate for the pressure error and the interfacial error pressure, using (3.40) there exists $\mathbf{v}_{h} \in X_{h}$ such that

$$
\begin{aligned}
\left\|p_{h}-q_{h}\right\|_{M}+ & \left\|\lambda_{h}-\zeta_{h}\right\|_{1 H^{1 / 2}(\Gamma)}+\left\|\boldsymbol{\beta}_{h}-\varrho_{h}\right\|_{\mathbb{R}^{2}} \\
\leq & \frac{C_{c}}{2} \frac{b\left(\mathbf{v}_{h},\left(p_{h}-q_{h}\right),\left(\boldsymbol{\beta}_{h}-\varrho_{h}\right)\right)-b_{I}\left(\mathbf{v}_{h},\left(\lambda_{h}-\zeta_{h}\right)\right)}{\left\|\mathbf{v}_{h}\right\|_{X}} \\
= & \frac{C_{c}}{2} \frac{b\left(\mathbf{v}_{h},\left(p-q_{h}\right),\left(\boldsymbol{\beta}-\varrho_{h}\right)\right)-b_{I}\left(\mathbf{v}_{h},\left(\lambda-\zeta_{h}\right)\right)}{\left\|\mathbf{v}_{h}\right\|_{X}} \\
& -\frac{C_{c}}{2} \frac{b\left(\mathbf{v}_{h},\left(p-p_{h}\right),\left(\boldsymbol{\beta}-\boldsymbol{\beta}_{h}\right)\right)-b_{I}\left(\mathbf{v}_{h},\left(\lambda-\lambda_{h}\right)\right)}{\left\|\mathbf{v}_{h}\right\|_{X}} \\
= & \frac{C_{c}}{2} \frac{b\left(\mathbf{v}_{h},\left(p-q_{h}\right),\left(\boldsymbol{\beta}-\varrho_{h}\right)\right)-b_{I}\left(\mathbf{v}_{h},\left(\lambda-\zeta_{h}\right)\right)}{\left\|\mathbf{v}_{h}\right\|_{X}}-\frac{C_{c}}{2} \frac{a_{\gamma}\left(\left(\mathbf{u}-\mathbf{u}_{h}\right), \mathbf{v}_{h}\right)}{\left\|\mathbf{v}_{h}\right\|_{X}} \\
\leq & C\left(\left\|p-q_{h}\right\|_{M}+\left\|\boldsymbol{\beta}-\varrho_{h}\right\|_{\mathbb{R}^{2}}+\left\|\lambda-\zeta_{h}\right\|_{1 H^{1 / 2}(\Gamma)}+\left\|\mathbf{u}-\mathbf{u}_{h}\right\|_{X}\right) .
\end{aligned}
$$

The error estimate (3.41) then follows from the triangle inequality, (3.51) and (3.50).

Remark: For $(\mathbf{u}, p)$ sufficiently smooth, $X_{f, h}, M_{f, h}, X_{p, h}, M_{p, h}$ given by (3.1)-(3.4), we have from $[4,15,28]$ that

$$
\begin{gathered}
\inf _{\mathbf{v}_{h} \in X_{f, h}}\left\|\mathbf{u}_{f}-\mathbf{v}_{h}\right\|_{X_{f}} \leq C h^{m}, \quad \inf _{q_{h} \in M_{f, h}}\left\|p_{f}-q_{h}\right\|_{1} L^{2}\left(\Omega_{f}\right) \leq C h^{m} \\
\inf _{\mathbf{v}_{h} \in X_{p, h}}\left\|\mathbf{u}_{p}-\mathbf{v}_{h}\right\|_{X_{p}} \leq C h^{k+1}, \quad \inf _{q_{h} \in M_{p, h}}\left\|p_{p}-q_{h}\right\|_{1} L^{2}\left(\Omega_{p}\right) \leq C h^{k+1} .
\end{gathered}
$$

Remark: For a sufficiently smooth function $\lambda$, the interpolation results can be extended to obtain

$$
\inf _{\zeta_{h} \in L_{h}}\left\|\lambda-\zeta_{h}\right\|_{1} H^{s}(\Gamma) \leq C h^{l+1-s}, s=0,1,
$$

and then by operator interpolation to yield

$$
\inf _{\zeta_{h} \in L_{h}}\left\|\lambda-\zeta_{h}\right\|_{1 H^{1 / 2}(\Gamma)} \leq C h^{l+1 / 2} .
$$

\section{Numerical Experiments}

In this section we numerically investigate the approximation of two, cylindrically symmetric, coupled Stokes-Darcy flow problems. The first experiment is performed on an example with a known solution. Rates of convergence of the approximation to the known solution are computed for several different choices of approximating elements and compared with those predicted by Theorem 3 . The second example we investigate is that of fluid flow through the eye. For this example we compare the flow profiles obtained assuming a parabolic inflow and outflow profile with that from using the defective boundary condition discussed above. 
In describing the approximation spaces/approximating elements below, we use $P_{k}$ to denote the space of polynomials of degree $\leq k$ on each triangle, which are continuous over the domain. The notation $\operatorname{discP}_{k}$ refers to the approximation spaces/approximating elements which are polynomials of degree $\leq k$ on each triangle, and are not required to be continuous over the domain.

For all the computations presented below the value used for $\gamma$ in (3.13) was $\gamma=1.0$.

\subsection{Example 1}

For this example we take $\Omega=(0,1 / 2) \times(-1 / 2,1 / 2), \Omega_{f}=(0,1 / 2) \times(0,1 / 2), \Omega_{p}=(0,1 / 2) \times$ $(-1 / 2,0)$, and $\Gamma=(0,1 / 2) \times\{0\}$. For the fluid velocity in $\Omega_{f}$ and $\Omega_{p}$ we use

$$
\mathbf{u}_{f}(r, z)=\mathbf{u}_{p}(r, z)=\left[\begin{array}{c}
-r \cos (\pi r) \sin (\pi z) \\
-\frac{2}{\pi} \cos (\pi r) \cos (\pi z)+r \sin (\pi r) \cos (\pi z)
\end{array}\right]
$$

and for the pressure in $\Omega_{f}$ and $\Omega_{p}$

$$
p_{f}=p_{p}=\sin (\pi z)(-\cos (\pi r)+2 \pi r \sin (\pi r))+4 r e^{-4 r} \cos (\pi z)-\frac{2}{\pi}\left(1-5 e^{-2}\right) .
$$

In addition we use $\nu=\nu_{\text {eff }}=1, \kappa=1$, and $\alpha_{a s}=1$.

Computations were performed on a series of meshes. Illustrated in Figure 4.1 is the computational mesh corresponding to mesh parameter $h=1 / 4$. The computed flow field and contour lines for the magnitude of the velocity on the mesh $h=1 / 8$, using Taylor-Hood $P_{2}-P_{1}$ elements for approximating $\left(\mathbf{u}_{f}, p_{f}\right)$, Raviart-Thomas $R T_{1}-\operatorname{disc} P_{1}$ elements for approximating $\left(\mathbf{u}_{p}, p_{p}\right)$, and $P_{1}$ elements for approximating the interfacial pressure $\lambda$, are given in Figures 4.2 and 4.3.

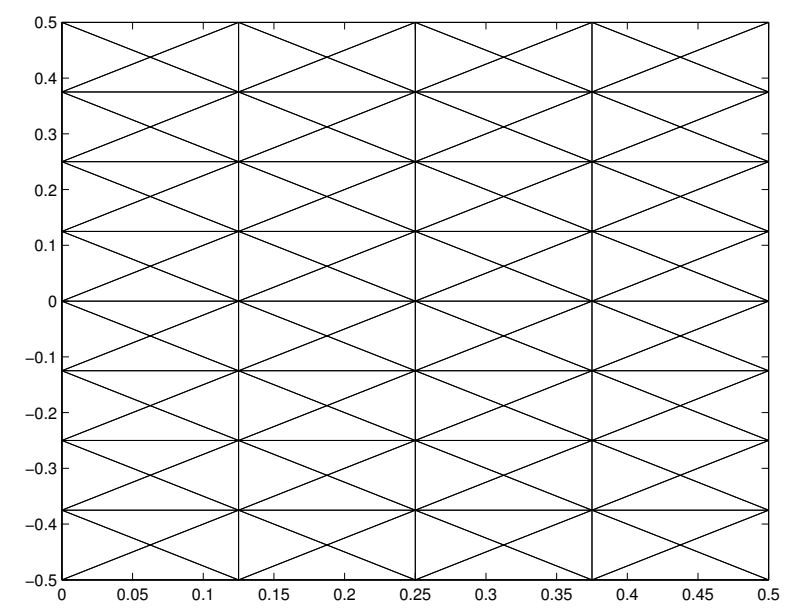

Figure 4.1: Computational mesh corresponding to $h=1 / 4$.

Presented in Table 4.1 are computations obtained using the mini-element approximation pair, $\left(P_{1}+\right.$ Bubble $)-P_{1}$ for $\left(\mathbf{u}_{f, h}, p_{f, h}\right)$, Raviart-Thomas $R T_{1}-\operatorname{disc} P_{1}$ for $\left(\mathbf{u}_{p, h}, p_{p, h}\right)$, and $P_{1}$ approximation 


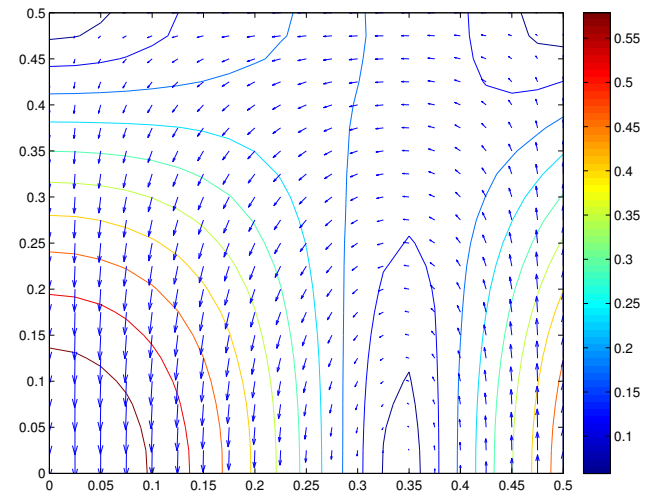

Figure 4.2: Computed Stokes velocity flow field for Example 1.

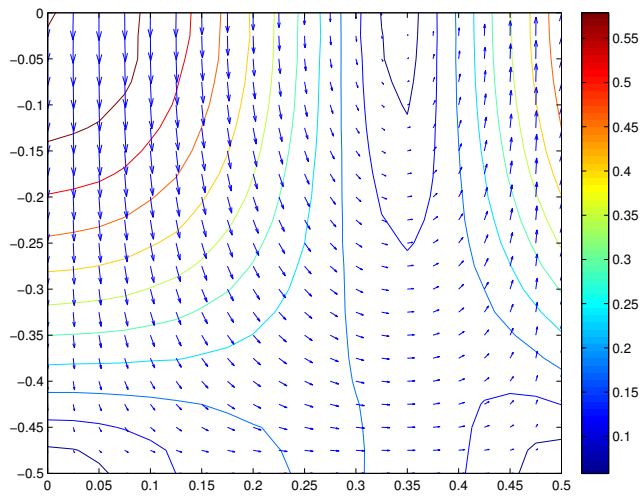

Figure 4.3: Computed Darcy velocity flow field for Example 1.

elements for $\lambda_{h}$. Theorem 3 predicts (bounded by the $i n f_{\mathbf{v}_{f, h} \in X_{f, h}}\left\|\mathbf{u}_{f}-\mathbf{v}_{f, h}\right\|_{1^{1}\left(\Omega_{f}\right)}$ term)

$\left\|\mathbf{u}_{f}-\mathbf{u}_{f, h}\right\|_{X_{f}}+\left\|p_{f}-p_{f, h}\right\|_{1 L^{2}\left(\Omega_{f}\right)}+\left\|\mathbf{u}_{p}-\mathbf{u}_{p, h}\right\|_{X_{p}}+\left\|p_{p}-p_{p, h}\right\|_{L^{2}\left(\Omega_{p}\right)}+\left\|\lambda-\lambda_{h}\right\|_{1 H^{1 / 2}(\Gamma)} \leq C h^{1}$.

The experimental convergence rates computed in Table 4.1 are consistent with (4.3).

Remark: As $L_{h} \subset{ }_{1} H^{1 / 2}(\Gamma)$, for a conforming method, we require that our approximation for $\lambda, \lambda_{h}$, be a continuous function. Assumption A1, $L_{h} \subset\left\{\left.\mathbf{v} \cdot \mathbf{n}_{p}\right|_{\Gamma}: \mathbf{v} \in X_{p, h}\right\}$, then implies that $R T_{0}-\operatorname{disc} P_{0}$ is not an appropriate choice as an approximation pair for $\left(\mathbf{u}_{p, h}, p_{p, h}\right)$.

Table 4.2 contains the computations obtained using Taylor-Hood $P_{2}-P_{1}$ approximating elements for $\left(\mathbf{u}_{f, h}, p_{f, h}\right)$, Raviart-Thomas $R T_{1}-\operatorname{disc} P_{1}$ for $\left(\mathbf{u}_{p, h}, p_{p, h}\right)$, and $P_{1}$ approximation elements for $\lambda_{h}$. Theorem 3 predicts (bounded by the $i n f_{\zeta_{h} \in L_{h}}\left\|\lambda-\zeta_{h}\right\|_{1^{1 / 2}(\Gamma)}$ term)

$\left\|\mathbf{u}_{f}-\mathbf{u}_{f, h}\right\|_{X_{f}}+\left\|p_{f}-p_{f, h}\right\|_{1 L^{2}\left(\Omega_{f}\right)}+\left\|\mathbf{u}_{p}-\mathbf{u}_{p, h}\right\|_{X_{p}}+\left\|p_{p}-p_{p, h}\right\|_{1 L^{2}\left(\Omega_{p}\right)}+\left\|\lambda-\lambda_{h}\right\|_{1^{1 / 2}(\Gamma)} \leq C h^{3 / 2}$.

The experimental convergence rates computed in Table 4.2 are consistent with (4.4).

The computations in Tables 4.3 and 4.4 were obtained using Taylor-Hood $P_{2}-P_{1}$ approximating elements for $\left(\mathbf{u}_{f, h}, p_{f, h}\right)$ and Raviart-Thomas $R T_{2}-d i s c P_{2}$ for $\left(\mathbf{u}_{p, h}, p_{p, h}\right)$. For Table $4.3, \lambda$ was approximated using $P_{1}$ elements, and for Table $4.4, \lambda$ was approximated using $P_{2}$ elements. For the results in Table 4.3, Theorem 3 predicts (bounded by the $i n f_{\zeta_{h} \in L_{h}}\left\|\lambda-\zeta_{h}\right\|_{1 H^{1 / 2}(\Gamma)}$ term)

$\left\|\mathbf{u}_{f}-\mathbf{u}_{f, h}\right\|_{X_{f}}+\left\|p_{f}-p_{f, h}\right\|_{1 L^{2}\left(\Omega_{f}\right)}+\left\|\mathbf{u}_{p}-\mathbf{u}_{p, h}\right\|_{X_{p}}+\left\|p_{p}-p_{p, h}\right\|_{1 L^{2}\left(\Omega_{p}\right)}+\left\|\lambda-\lambda_{h}\right\|_{1 H^{1 / 2}(\Gamma)} \leq C h^{3 / 2}$,

and for the results in Table 4.4, Theorem 3 predicts (bounded by the $i n f_{\mathbf{v}_{f, h} \in X_{f, h}}\left\|\mathbf{u}_{f}-\mathbf{v}_{f, h}\right\|_{1 H^{1}\left(\Omega_{f}\right)}$ and $i n f_{q_{f, h} \in M_{f, h}}\left\|p_{f}-q_{f, h}\right\|_{1 L^{2}\left(\Omega_{f}\right)}$ term)

$\left\|\mathbf{u}_{f}-\mathbf{u}_{f, h}\right\|_{X_{f}}+\left\|p_{f}-p_{f, h}\right\|_{1 L^{2}\left(\Omega_{f}\right)}+\left\|\mathbf{u}_{p}-\mathbf{u}_{p, h}\right\|_{X_{p}}+\left\|p_{p}-p_{p, h}\right\|_{1 L^{2}\left(\Omega_{p}\right)}+\left\|\lambda-\lambda_{h}\right\|_{1 H^{1 / 2}(\Gamma)} \leq C h^{2}$,

The experimental convergence rates computed in Tables 4.34 .4 are consistent with (4.5) and (4.6), respectively. 


\begin{tabular}{|c||c|c||c|c||c|c|}
\hline \multicolumn{1}{|c|}{$h$} & $\left\|\mathbf{u}_{f}-\mathbf{u}_{f, h}\right\|_{X_{f}}$ & Cvg. rate & $\left\|\mathbf{u}_{f}-\mathbf{u}_{f, h}\right\|_{L^{2}\left(\Omega_{f}\right)}$ & Cvg. rate & $\left\|p_{f}-p_{f, h}\right\|_{L^{2}\left(\Omega_{f}\right)}$ & Cvg. rate \\
\hline $1 / 2$ & $1.541 \mathrm{E}-01$ & & $4.705 \mathrm{E}-03$ & & $9.894 \mathrm{E}-02$ & \\
$1 / 4$ & $7.513 \mathrm{E}-02$ & 1.04 & $1.230 \mathrm{E}-03$ & 1.94 & $3.141 \mathrm{E}-02$ & 1.66 \\
$1 / 8$ & $3.708 \mathrm{E}-02$ & 1.02 & $3.106 \mathrm{E}-04$ & 1.98 & $1.059 \mathrm{E}-02$ & 1.57 \\
$1 / 12$ & $2.460 \mathrm{E}-02$ & 1.01 & $1.384 \mathrm{E}-04$ & 1.99 & $5.619 \mathrm{E}-03$ & 1.56 \\
$1 / 16$ & $1.841 \mathrm{E}-02$ & 1.01 & $7.791 \mathrm{E}-05$ & 2.00 & $3.593 \mathrm{E}-03$ & 1.55 \\
\hline \hline
\end{tabular}

\begin{tabular}{|c|c|c|c|c|c|c|}
\hline \multicolumn{7}{|c|}{ Darcy flow approximation errors } \\
\hline$h$ & $\left\|\mathbf{u}_{p}-\mathbf{u}_{p, h}\right\|_{X_{p}}$ & Cvg. rate & $\left\|\mathbf{u}_{p}-\mathbf{u}_{p, h}\right\|_{1 L^{2}\left(\Omega_{p}\right)}$ & Cvg. rate & $\left\|p_{p}-p_{p, h}\right\|_{1 L^{2}\left(\Omega_{p}\right)}$ & Cvg. rate \\
\hline $1 / 2$ & 8.967E-03 & & $7.424 \mathrm{E}-03$ & & $2.989 \mathrm{E}-02$ & \\
\hline $1 / 4$ & $2.365 \mathrm{E}-03$ & 1.92 & $2.030 \mathrm{E}-03$ & 1.87 & $6.569 \mathrm{E}-03$ & 2.19 \\
\hline $1 / 8$ & $6.230 \mathrm{E}-04$ & 1.92 & $5.405 \mathrm{E}-04$ & 1.91 & $1.594 \mathrm{E}-03$ & 2.04 \\
\hline $1 / 12$ & $2.703 \mathrm{E}-04$ & 2.06 & $2.314 \mathrm{E}-04$ & 2.09 & 7.023E-04 & 2.02 \\
\hline $1 / 16$ & $1.468 \mathrm{E}-04$ & 2.12 & $1.232 \mathrm{E}-04$ & 2.19 & $3.932 \mathrm{E}-04$ & 2.02 \\
\hline
\end{tabular}

\begin{tabular}{|c|c|c|c|c|c|c|}
\hline \multicolumn{5}{|c|}{ Interfacial pressure approximation errors } & & \\
\hline$h$ & $\left\|\lambda-\lambda_{h}\right\|_{1} H^{1}(\Gamma)$ & Cvg. rate & $\left\|\lambda-\lambda_{h}\right\|_{1} L^{2}(\Gamma)$ & Cvg. rate & & \\
\hline $1 / 2$ & $1.508 \mathrm{E}-01$ & & $4.107 \mathrm{E}-02$ & & & \\
\hline $1 / 4$ & 8.033E-02 & 0.91 & 8.929E-03 & 2.20 & & \\
\hline $1 / 8$ & 4.213E-02 & 0.93 & $2.161 \mathrm{E}-03$ & 2.05 & & \\
\hline $1 / 12$ & $2.845 \mathrm{E}-02$ & 0.97 & $9.513 \mathrm{E}-04$ & 2.02 & & \\
\hline $1 / 16$ & $2.145 \mathrm{E}-02$ & 0.98 & $5.325 \mathrm{E}-04$ & 2.02 & & \\
\hline
\end{tabular}

Table 4.1: Example 1 using $\left(P_{1}+\right.$ Bubble $)-P_{1}, R T_{1}-\operatorname{disc} P_{1}$, and $P_{1}$ approximation elements.

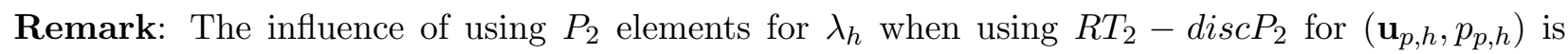
clearly demonstrated by comparing the numerical results in Tables 4.3 and 4.4.

\subsection{Example 2}

To demonstrate the difference between flow fields generated assuming a parabolic inflow and outflow profile with that from the defective boundary conditions given by (2.3) and (2.7), we simulate fluid flow in the eye. In this simulation we assume the eye is looking straight up. Fluid in the eye is generated by the ciliary body which is located on the wall of the eye adjacent to the lens [31]. Fluid flows from the ciliary body into the Anterior Cavity (front section of the eye), AC, by passing between the lens and the iris and then flowing through the pupil, see Figure 4.5. We assume a flow rate of $2 \mu \mathrm{l} / \mathrm{min}$ [24]. The fluid exits the AC through the Trabecular Meshwork, TM, located on the wall of the eye slightly above where the iris attaches to the wall. After flowing through the TM the fluids enters the Canal of Schlemm. The model geometry of the eye illustrated in Figure 4.5 was constructed using $[12,13]$ and [35]. For the model we assume:

1. The radius of the inside of the cornea is $7.2 \mathrm{~mm}$.

2. The radius of the lens is $12.5 \mathrm{~mm}$.

3. The distance between the lens and the cornea along the vertical axis is $2.7 \mathrm{~mm}$. 


\begin{tabular}{|c||c|c||c|c||c|c|}
\hline \multicolumn{1}{|c|}{$h$} & $\left\|\mathbf{u}_{f}-\mathbf{u}_{f, h}\right\|_{X_{f}}$ & Cvg. rate & $\left\|\mathbf{u}_{f}-\mathbf{u}_{f, h}\right\|_{L^{2}\left(\Omega_{f}\right)}$ & Cvg. rate & $\left\|p_{f}-p_{f, h}\right\|_{L^{2}\left(\Omega_{f}\right)}$ & Cvg. rate \\
\hline $1 / 2$ & $1.761 \mathrm{E}-02$ & & $5.184 \mathrm{E}-04$ & & $1.450 \mathrm{E}-02$ & \\
$1 / 4$ & $4.452 \mathrm{E}-03$ & 1.98 & $6.693 \mathrm{E}-05$ & 2.95 & $3.265 \mathrm{E}-03$ & 2.15 \\
$1 / 8$ & $1.115 \mathrm{E}-03$ & 2.00 & $8.390 \mathrm{E}-06$ & 3.00 & $7.836 \mathrm{E}-04$ & 2.06 \\
$1 / 12$ & $4.956 \mathrm{E}-04$ & 2.00 & $2.486 \mathrm{E}-06$ & 3.00 & $3.454 \mathrm{E}-04$ & 2.02 \\
$1 / 16$ & $2.788 \mathrm{E}-04$ & 2.00 & $1.048 \mathrm{E}-06$ & 3.00 & $1.937 \mathrm{E}-04$ & 2.01 \\
\hline \hline
\end{tabular}

\begin{tabular}{|c||c|c||c|c||c|c|}
\hline \hline \multicolumn{7}{|c|}{} \\
$h$ & $\left\|\mathbf{u}_{p}-\mathbf{u}_{p, h}\right\|_{X_{p}}$ & Cvg. rate & $\left\|\mathbf{u}_{p}-\mathbf{u}_{p, h}\right\|_{1 L^{2}\left(\Omega_{p}\right)}$ & Cvg. rate & $\left\|p_{p}-p_{p, h}\right\|_{1 L^{2}\left(\Omega_{p}\right)}$ & Cvg. rate \\
\hline $1 / 2$ & $8.966 \mathrm{E}-03$ & & $7.415 \mathrm{E}-03$ & & $7.880 \mathrm{E}-03$ & \\
$1 / 4$ & $2.344 \mathrm{E}-03$ & 1.94 & $2.007 \mathrm{E}-03$ & 1.89 & $2.032 \mathrm{E}-03$ & 1.96 \\
$1 / 8$ & $6.164 \mathrm{E}-04$ & 1.93 & $5.331 \mathrm{E}-04$ & 1.91 & $5.140 \mathrm{E}-04$ & 1.98 \\
$1 / 12$ & $2.671 \mathrm{E}-04$ & 2.06 & $2.277 \mathrm{E}-04$ & 2.10 & $2.291 \mathrm{E}-04$ & 1.99 \\
$1 / 16$ & $1.449 \mathrm{E}-04$ & 2.13 & $1.210 \mathrm{E}-04$ & 2.20 & $1.290 \mathrm{E}-04$ & 2.00 \\
\hline \hline
\end{tabular}

\begin{tabular}{|c|c|c|c|c|c|c|}
\hline \multicolumn{5}{|c|}{ Interfacial pressure approximation errors } & & \\
\hline$h$ & $\left\|\lambda-\lambda_{h}\right\|_{1} H^{1}(\Gamma)$ & Cvg. rate & $\left\|\lambda-\lambda_{h}\right\|_{1} L^{2}(\Gamma)$ & Cvg. rate & & \\
\hline $1 / 2$ & $1.451 \mathrm{E}-01$ & & $4.978 \mathrm{E}-03$ & & & \\
\hline $1 / 4$ & 7.995E-02 & 0.86 & $1.286 \mathrm{E}-03$ & 1.95 & & \\
\hline $1 / 8$ & $4.210 \mathrm{E}-02$ & 0.93 & $3.390 \mathrm{E}-04$ & 1.92 & & \\
\hline $1 / 12$ & $2.844 \mathrm{E}-02$ & 0.97 & $1.529 \mathrm{E}-04$ & 1.96 & & \\
\hline $1 / 16$ & $2.145 \mathrm{E}-02$ & 0.98 & $8.652 \mathrm{E}-05$ & 1.98 & & \\
\hline
\end{tabular}

Table 4.2: Example 1 using $P_{2}-P_{1}, R T_{1}-\operatorname{disc} P_{1}$, and $P_{1}$ approximation elements.

4. The pupil aperture is $3 \mathrm{~mm}$.

5. The lower side of the iris has the same curvature as the lens. The top side of the iris is approximated as a straight line. The width of the iris is approximately $0.5 \mathrm{~mm}$, and we assume the iris attaches to the cornea. (Physically the iris attaches to the ciliary muscles very near the cornea.)

6. The gap between the iris and the lens is $0.25 \mathrm{~mm}$.

7. The length of the interface between the $\mathrm{AC}$ and $\mathrm{TM}$ is $0.6 \mathrm{~mm}$.

8. The width of the TM at the bottom is $0.1 \mathrm{~mm}$.

9. The length of the interface of the TM with the Canal of Schlemm is $0.3 \mathrm{~mm}$.

10. A straight line connects the point at the top of the interface of the AC and TM with the point at the top of the interface of the TM with the Canal of Schlemm.

The fluid flow in the AC is modeling using the Stokes equations and that through the TM modeled using the Darcy equations. For the kinematic viscosity of the fluid we use $\nu=0.66 \mathrm{~mm} / \mathrm{s}$ (approximately that of water), and in the TM for the effective viscosity $\nu_{\text {eff }}=0.66 \mathrm{~mm} / \mathrm{s}$, and for the permeability $\kappa=2.0 \times 10^{-6} \mathrm{~mm}^{2}[2]$. 


\begin{tabular}{|c||c|c||c|c||c|c|}
\hline \multicolumn{1}{|c|}{$h$} & $\left\|\mathbf{u}_{f}-\mathbf{u}_{f, h}\right\|_{X_{f}}$ & Cvg. rate & $\left\|\mathbf{u}_{f}-\mathbf{u}_{f, h}\right\|_{L^{2}\left(\Omega_{f}\right)}$ & Cvg. rate & $\left\|p_{f}-p_{f, h}\right\|_{L^{2}\left(\Omega_{f}\right)}$ & Cvg. rate \\
\hline $1 / 2$ & $1.761 \mathrm{E}-02$ & & $5.185 \mathrm{E}-04$ & & $1.451 \mathrm{E}-02$ & \\
$1 / 4$ & $4.452 \mathrm{E}-03$ & 1.98 & $6.692 \mathrm{E}-05$ & 2.95 & $3.264 \mathrm{E}-03$ & 2.15 \\
$1 / 8$ & $1.115 \mathrm{E}-03$ & 2.00 & $8.389 \mathrm{E}-06$ & 3.00 & $7.836 \mathrm{E}-04$ & 2.06 \\
$1 / 12$ & $4.956 \mathrm{E}-04$ & 2.00 & $2.485 \mathrm{E}-06$ & 3.00 & $3.454 \mathrm{E}-04$ & 2.02 \\
$1 / 16$ & $2.788 \mathrm{E}-04$ & 2.00 & $1.048 \mathrm{E}-06$ & 3.00 & $1.937 \mathrm{E}-04$ & 2.01 \\
\hline \hline
\end{tabular}

\begin{tabular}{|c||c|c||c|c||c|c|}
\hline \hline \multicolumn{7}{|c|}{} \\
$h$ & $\left\|\mathbf{u}_{p}-\mathbf{u}_{p, h}\right\|_{X_{p}}$ & Cvg. rate & $\left\|\mathbf{u}_{p}-\mathbf{u}_{p, h}\right\|_{1 L^{2}\left(\Omega_{p}\right)}$ & Cvg. rate & $\left\|p_{p}-p_{p, h}\right\|_{1 L^{2}\left(\Omega_{p}\right)}$ & Cvg. rate \\
\hline $1 / 2$ & $2.088 \mathrm{E}-02$ & & $2.084 \mathrm{E}-02$ & & $1.277 \mathrm{E}-03$ & \\
$1 / 4$ & $8.199 \mathrm{E}-03$ & 1.35 & $8.177 \mathrm{E}-03$ & 1.35 & $1.708 \mathrm{E}-04$ & 2.90 \\
$1 / 8$ & $3.143 \mathrm{E}-03$ & 1.38 & $3.129 \mathrm{E}-03$ & 1.39 & $2.532 \mathrm{E}-05$ & 2.75 \\
$1 / 12$ & $1.751 \mathrm{E}-03$ & 1.44 & $1.743 \mathrm{E}-03$ & 1.44 & $9.069 \mathrm{E}-06$ & 2.53 \\
$1 / 16$ & $1.149 \mathrm{E}-03$ & 1.47 & $1.144 \mathrm{E}-03$ & 1.46 & $4.536 \mathrm{E}-06$ & 2.41 \\
\hline
\end{tabular}

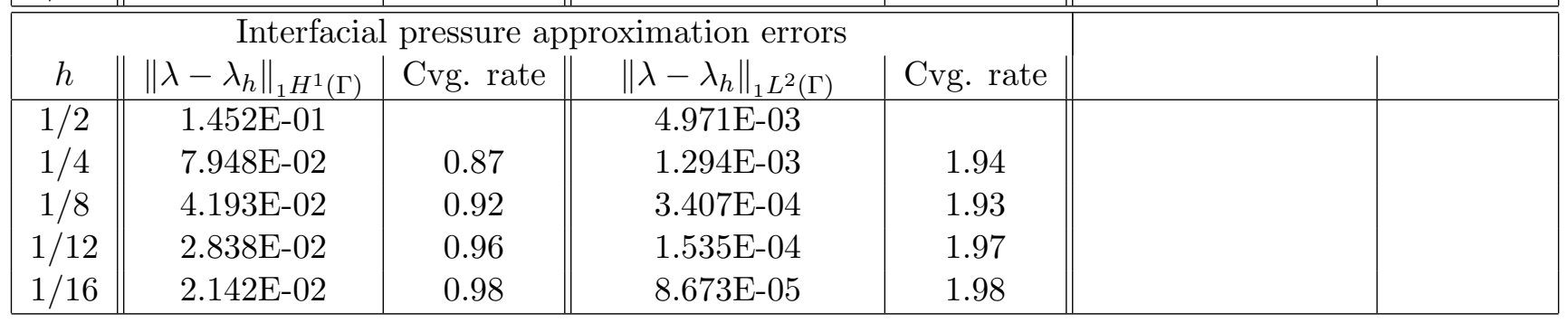

Table 4.3: Example 1 using $P_{2}-P_{1}, R T_{2}-\operatorname{disc} P_{2}$, and $P_{1}$ approximation elements.

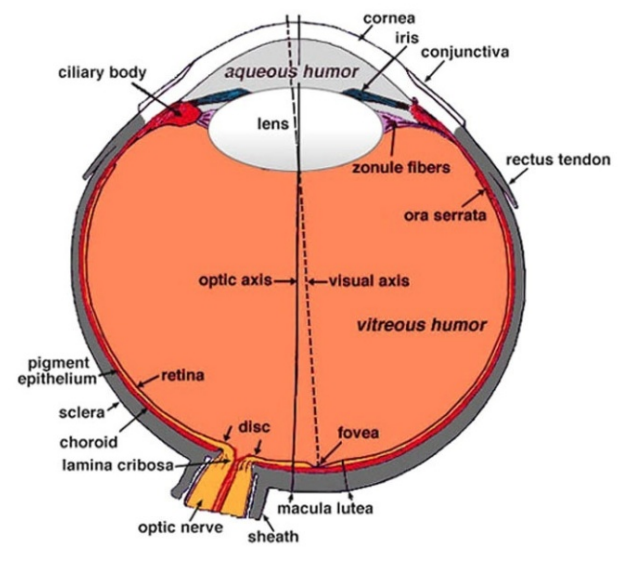

Figure 4.4: Illustration of the anatomy of an eye $[25]$.

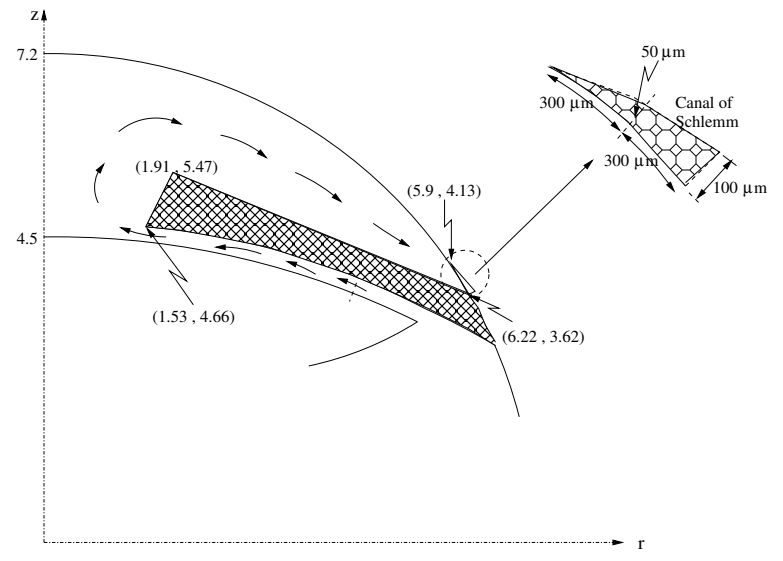

Figure 4.5: Model for simulating fluid flow through the eye.

In our modeling equations there arises from the Beavers-Joseph-Saffman boundary condition (2.10) a frictional constant, denoted by $\alpha_{a s}$ in (2.21). As we do not have a reference value for $\alpha_{a s}$, we use the nominal value $\alpha_{a s}=1$ in our computations.

Computations were performed on a series of three meshes. The initial mesh $(h=1)$ is given in Figures 4.6 and 4.7. The two subsequent meshes $(h=1 / 2, h=1 / 4)$ were obtained by mid edge 


\begin{tabular}{|c||c|c||c|c||c|c|}
\hline \multicolumn{1}{|c||}{} & $\left\|\mathbf{u}_{f}-\mathbf{u}_{f, h}\right\|_{X_{f}}$ & Cvg. rate & $\left\|\mathbf{u}_{f}-\mathbf{u}_{f, h}\right\|_{1^{2}\left(\Omega_{f}\right)}$ & Cvg. rate & $\left\|p_{f}-p_{f, h}\right\|_{1^{2}\left(\Omega_{f}\right)}$ & Cvg. rate \\
\hline $1 / 2$ & $1.761 \mathrm{E}-02$ & & $5.163 \mathrm{E}-04$ & & $1.450 \mathrm{E}-02$ & \\
$1 / 4$ & $4.452 \mathrm{E}-03$ & 1.98 & $6.686 \mathrm{E}-05$ & 2.95 & $3.264 \mathrm{E}-03$ & 2.15 \\
$1 / 8$ & $1.115 \mathrm{E}-03$ & 2.00 & $8.387 \mathrm{E}-06$ & 2.99 & $7.835 \mathrm{E}-04$ & 2.06 \\
$1 / 12$ & $4.955 \mathrm{E}-04$ & 2.00 & $2.485 \mathrm{E}-06$ & 3.00 & $3.454 \mathrm{E}-04$ & 2.02 \\
$1 / 16$ & $2.788 \mathrm{E}-04$ & 2.00 & $1.048 \mathrm{E}-06$ & 3.00 & $1.937 \mathrm{E}-04$ & 2.01 \\
$1 / 20$ & $1.784 \mathrm{E}-04$ & 2.00 & $5.367 \mathrm{E}-07$ & 3.00 & $1.238 \mathrm{E}-04$ & 2.01 \\
\hline \hline
\end{tabular}

\begin{tabular}{|c|c|c|c|c|c|c|}
\hline \multicolumn{7}{|c|}{ Darcy flow approximation errors } \\
\hline$h$ & $\left\|\mathbf{u}_{p}-\mathbf{u}_{p, h}\right\|_{X_{p}}$ & Cvg. rate & $\left\|\mathbf{u}_{p}-\mathbf{u}_{p, h}\right\|_{1} L^{2}\left(\Omega_{p}\right)$ & Cvg. rate & $\left\|p_{p}-p_{p, h}\right\|_{1 L^{2}\left(\Omega_{p}\right)}$ & Cvg. rate \\
\hline $1 / 2$ & $3.082 \mathrm{E}-03$ & & $3.029 \mathrm{E}-03$ & & $1.143 \mathrm{E}-03$ & \\
\hline $1 / 4$ & $8.579 \mathrm{E}-04$ & 1.85 & 8.413E-04 & 1.85 & $1.304 \mathrm{E}-04$ & 3.13 \\
\hline $1 / 8$ & $1.837 \mathrm{E}-04$ & 2.22 & $1.806 \mathrm{E}-04$ & 2.22 & $1.474 \mathrm{E}-05$ & 3.14 \\
\hline $1 / 12$ & $7.053 \mathrm{E}-05$ & 2.36 & $6.954 \mathrm{E}-05$ & 2.35 & $4.219 \mathrm{E}-06$ & 3.09 \\
\hline $1 / 16$ & $3.527 \mathrm{E}-05$ & 2.41 & $3.483 \mathrm{E}-05$ & 2.40 & $1.751 \mathrm{E}-06$ & 3.06 \\
\hline $1 / 20$ & 2.049E-05 & 2.43 & $2.026 \mathrm{E}-05$ & 2.43 & 8.877E-07 & 3.04 \\
\hline
\end{tabular}

\begin{tabular}{|c|c|c|c|c|c|c|}
\hline \multicolumn{5}{|c|}{ Interfacial pressure approximation errors } & & \\
\hline$h$ & $\left\|\lambda-\lambda_{h}\right\|_{1} H^{1}(\Gamma)$ & Cvg. rate & $\left\|\lambda-\lambda_{h}\right\|_{1} L^{2}(\Gamma)$ & Cvg. rate & & \\
\hline $1 / 2$ & $3.470 \mathrm{E}-02$ & & $1.412 \mathrm{E}-03$ & & & \\
\hline $1 / 4$ & $9.454 \mathrm{E}-03$ & 1.88 & 1.819E-04 & 2.96 & & \\
\hline $1 / 8$ & $2.441 \mathrm{E}-03$ & 1.95 & $2.314 \mathrm{E}-05$ & 2.97 & & \\
\hline $1 / 12$ & $1.091 \mathrm{E}-03$ & 1.99 & $6.933 \mathrm{E}-06$ & 2.97 & & \\
\hline $1 / 16$ & $6.145 \mathrm{E}-04$ & 2.00 & $2.941 \mathrm{E}-06$ & 2.98 & & \\
\hline $1 / 20$ & $3.934 \mathrm{E}-04$ & 2.00 & $1.510 \mathrm{E}-06$ & 2.99 & & \\
\hline
\end{tabular}

Table 4.4: Example 1 using $P_{2}-P_{1}, R T_{2}-\operatorname{disc} P_{2}$, and $P_{2}$ approximation elements.

refinement, whereby each triangle in the mesh was divided into four smaller triangles by connecting the mid edges of each side.

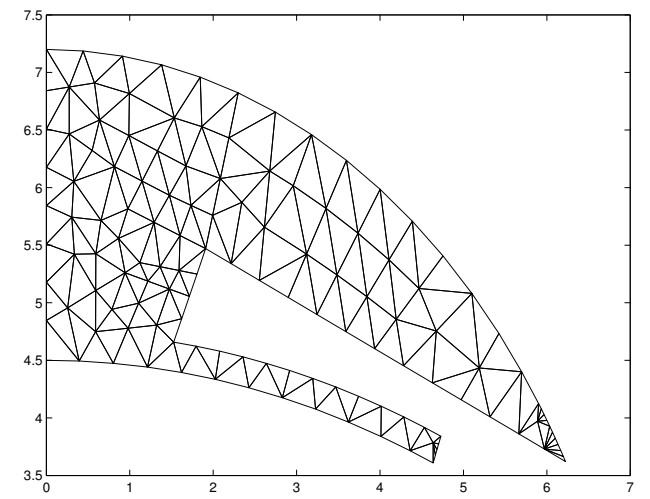

Figure 4.6: Computed mesh for the AC corresponding to $h=1$.

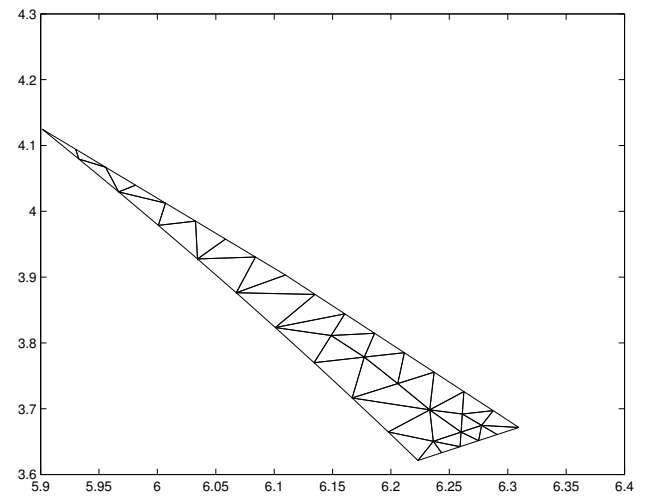

Figure 4.7: Computed mesh for the TM corresponding to $h=1$. 
The approximating elements used were the Taylor-Hood $P_{2}-P_{1}$ for the velocity-pressure approximation in the $\mathrm{AC}$, Raviart-Thomas $R T_{1}-\operatorname{disc}_{1}$ for the velocity-pressure in the TM, and continuous, piecewise linear elements, $P_{1}$, for the interfacial pressure term along the interface between the AC and TM regions. Presented in Tables 4.5 and 4.6 are various norms of the approximation computed on the three different meshes for the case of the degenerate boundary condition.

\begin{tabular}{|c||c|c|c|}
\hline \multicolumn{4}{|c|}{ Stokes flow norms } \\
$h$ & $\left\|\mathbf{u}_{f, h}\right\|_{X_{f}}$ & $\left\|\mathbf{u}_{f, h}\right\|_{1 L^{2}\left(\Omega_{f}\right)}$ & $\left\|p_{f, h}\right\|_{1} L^{2}\left(\Omega_{f}\right)$ \\
\hline 1 & $1.717 \mathrm{E}-01$ & $1.616 \mathrm{E}-02$ & $3.977 \mathrm{E}+00$ \\
$1 / 2$ & $1.725 \mathrm{E}-01$ & $1.622 \mathrm{E}-02$ & $3.981 \mathrm{E}+00$ \\
$1 / 4$ & $1.726 \mathrm{E}-01$ & $1.623 \mathrm{E}-02$ & $3.981 \mathrm{E}+00$ \\
\hline \hline \multicolumn{4}{|c|}{ Darcy flow norms } \\
$h$ & $\left\|\mathbf{u}_{p, h}\right\|_{X_{p}}$ & $\left\|\mathbf{u}_{p, h}\right\|_{1 L^{2}\left(\Omega_{p}\right)}$ & $\left\|p_{p, h}\right\|_{1 L^{2}\left(\Omega_{p}\right)}$ \\
\hline 1 & $1.002 \mathrm{E}-03$ & $1.002 \mathrm{E}-03$ & $1.363 \mathrm{E}+01$ \\
$1 / 2$ & $9.967 \mathrm{E}-04$ & $9.967 \mathrm{E}-04$ & $1.353 \mathrm{E}+01$ \\
$1 / 4$ & $9.943 \mathrm{E}-04$ & $9.943 \mathrm{E}-04$ & $1.348 \mathrm{E}+01$ \\
\hline \hline \multicolumn{5}{|c|}{ Interfacial pressure norms } & \\
$h$ & $\left\|\lambda_{h}\right\|_{1} H^{1}(\Gamma)$ & $\left\|\lambda_{h}\right\|_{1} L^{2}(\Gamma)$ & \\
\hline 1 & $3.017 \mathrm{E}+01$ & $9.637 \mathrm{E}-01$ & \\
$1 / 2$ & $6.345 \mathrm{E}+01$ & $1.078 \mathrm{E}+00$ & \\
$1 / 4$ & $7.978 \mathrm{E}+01$ & $1.026 \mathrm{E}+00$ & \\
\hline
\end{tabular}

\begin{tabular}{|c||c|c|c|}
\hline \multicolumn{4}{|c|}{ Stokes flow norms } \\
$h$ & $\left\|\mathbf{u}_{f, h}\right\|_{X_{f}}$ & $\left\|\mathbf{u}_{f, h}\right\|_{1 L^{2}\left(\Omega_{f}\right)}$ & $\left\|p_{f, h}\right\|_{1 L^{2}\left(\Omega_{f}\right)}$ \\
\hline 1 & $1.732 \mathrm{E}-01$ & $1.620 \mathrm{E}-02$ & $4.003 \mathrm{E}+00$ \\
$1 / 2$ & $1.742 \mathrm{E}-01$ & $1.627 \mathrm{E}-02$ & $4.008 \mathrm{E}+00$ \\
$1 / 4$ & $1.743 \mathrm{E}-01$ & $1.628 \mathrm{E}-02$ & $4.009 \mathrm{E}+00$ \\
\hline \hline \multicolumn{4}{|c|}{ Darcy flow norms } \\
$h$ & $\left\|\mathbf{u}_{p, h}\right\|_{X_{p}}$ & $\left\|\mathbf{u}_{p, h}\right\|_{1 L^{2}\left(\Omega_{p}\right)}$ & $\left\|p_{p, h}\right\|_{1 L^{2}\left(\Omega_{p}\right)}$ \\
\hline 1 & $1.111 \mathrm{E}-03$ & $1.111 \mathrm{E}-03$ & $1.606 \mathrm{E}+01$ \\
$1 / 2$ & $1.111 \mathrm{E}-03$ & $1.111 \mathrm{E}-03$ & $1.607 \mathrm{E}+01$ \\
$1 / 4$ & $1.111 \mathrm{E}-03$ & $1.111 \mathrm{E}-03$ & $1.607 \mathrm{E}+01$ \\
\hline \hline \multicolumn{5}{|c|}{ Interfacial pressure norms } & \\
$h$ & $\left\|\lambda_{h}\right\|_{1} H^{1}(\Gamma)$ & $\left\|\lambda_{h}\right\|_{1} L^{2}(\Gamma)$ & \\
\hline 1 & $3.395 \mathrm{E}+01$ & $1.064 \mathrm{E}+00$ & \\
$1 / 2$ & $7.273 \mathrm{E}+01$ & $1.212 \mathrm{E}+00$ & \\
$1 / 4$ & $9.212 \mathrm{E}+01$ & $1.154 \mathrm{E}+00$ & \\
\hline
\end{tabular}

Table 4.5: Example 2 using $P_{2}-P_{1}, R T_{1}-$ $\operatorname{disc} P_{1}$, and $P_{1}$ approximation elements, with a defective boundary condition.

Table 4.6: Example 2 using $P_{2}-P_{1}, R T_{1}-$ disc $P_{1}$, and $P_{1}$ approximation elements, assuming a parabolic inflow and outflow profile.

Illustrated in Figures 4.8-4.11 and 4.12-4.15 are the flow fields and pressure plots obtained on the finest mesh, $h=1 / 4$, for the cases of the degenerate boundary condition and an assumed parabolic inflow and outflow profile, respectively. Visually the difference in the computed solutions is most apparent in the velocity and pressure plots in the TM, i.e. by comparing Figures 4.10, 4.11, with Figures 4.14, 4.15. Consistent with (2.26), for simulations generated using the defective boundary condition formulation we note that the pressure along the outflow boundary is constant, and the flow much more uniform than for the case when the parabolic profile for the outflow is imposed.

\section{References}

[1] F. Assous, P. Ciarlet, Jr., and S. Labrunie. Theoretical tools to solve the axisymmetric Maxwell equations. Math. Methods Appl. Sci., 25(1):49-78, 2002.

[2] R. Avtar and R. Srivastava. Modelling aqueous humor outflow through trabecular meshwork. Appl. Math. Comput., 189:734-745, 2007.

[3] G. Beavers and D. Joseph. Boundary conditions at a naturally impermeable wall. J. Fluid Mech., 30:197-207, 1967. 


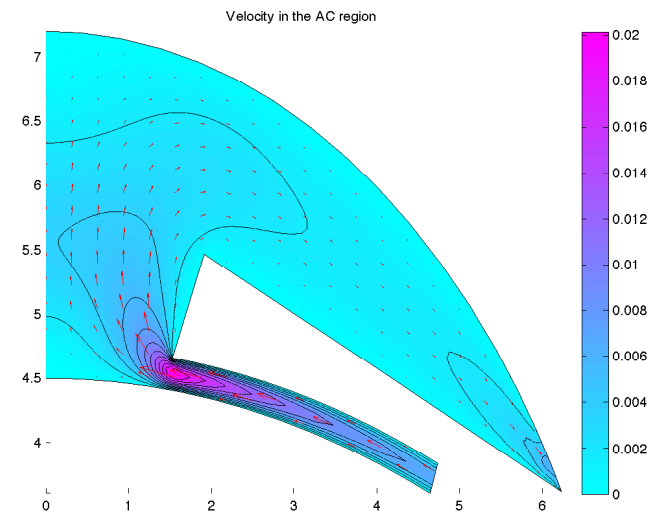

Figure 4.8: Computed flow field in the AC, $h=1 / 4$, for a defective boundary condition.

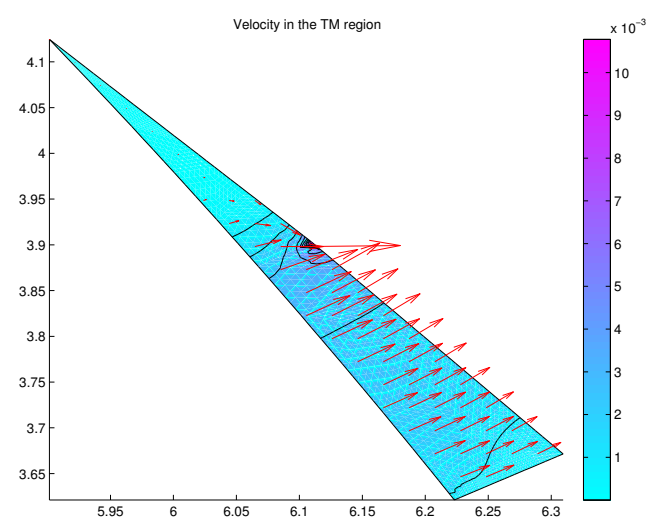

Figure 4.10: Computed flow field in the TM, $h=1 / 4$, for a defective boundary condition.

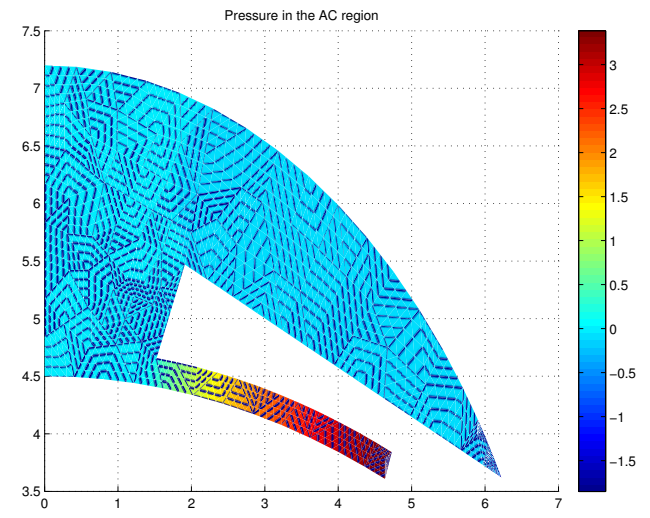

Figure 4.9: Computed pressure profile in the AC, $h=1 / 4$, for a defective boundary condition.

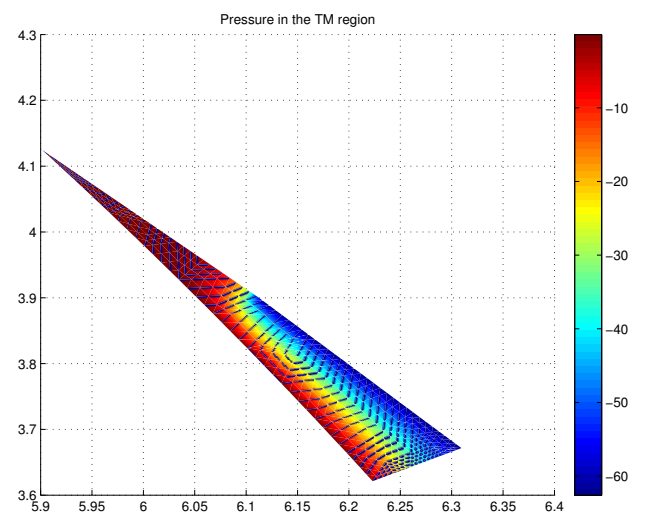

Figure 4.11: Computed pressure profile in the $\mathrm{TM}, h=1 / 4$, for a defective boundary condition.

[4] Z. Belhachmi, C. Bernardi, and S. Deparis. Weighted Clément operator and application to the finite element discretization of the axisymmetric Stokes problem. Numer. Math., 105(2):217$247,2006$.

[5] Z. Belhachmi, C. Bernardi, S. Deparis, and F. Hecht. A truncated Fourier/finite element discretization of the Stokes equations in an axisymmetric domain. Math. Models Methods Appl. Sci., 16(2):233-263, 2006.

[6] C. Bernardi, M. Dauge, and Y. Maday. Spectral methods for axisymmetric domains, volume 3 of Series in Applied Mathematics (Paris). Gauthier-Villars, 1999. Numerical algorithms and tests due to Mejdi Azaïez.

[7] F. Brezzi and M. Fortin. Mixed and hybrid finite element methods. Springer-Verlag, New York, 1991. 


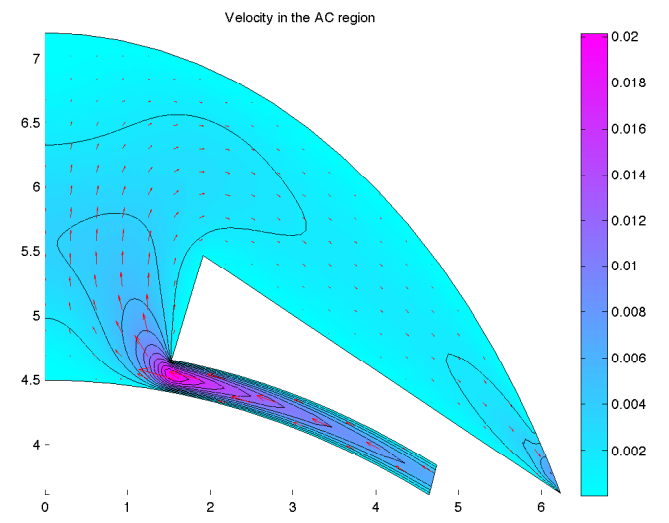

Figure 4.12: Computed flow field in the AC, $h=1 / 4$, for a parabolic inflow and outflow profile.

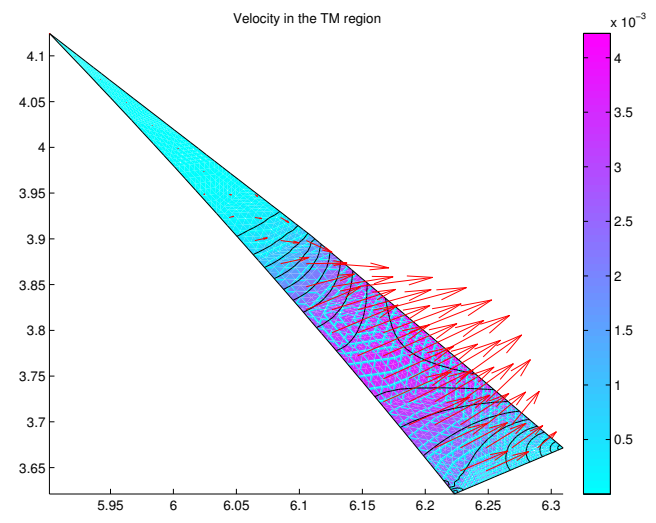

Figure 4.14: Computed flow field in the TM, $h=1 / 4$, for a parabolic inflow and outflow profile.

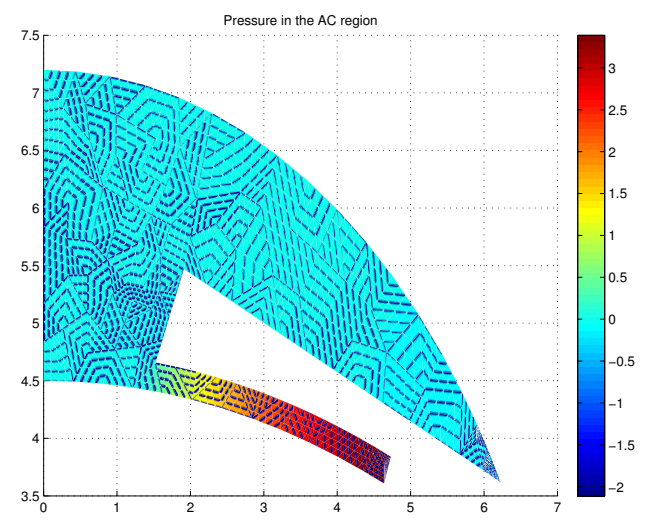

Figure 4.13: Computed pressure profile in the $\mathrm{AC}, h=1 / 4$, for a parabolic inflow and outflow profile.

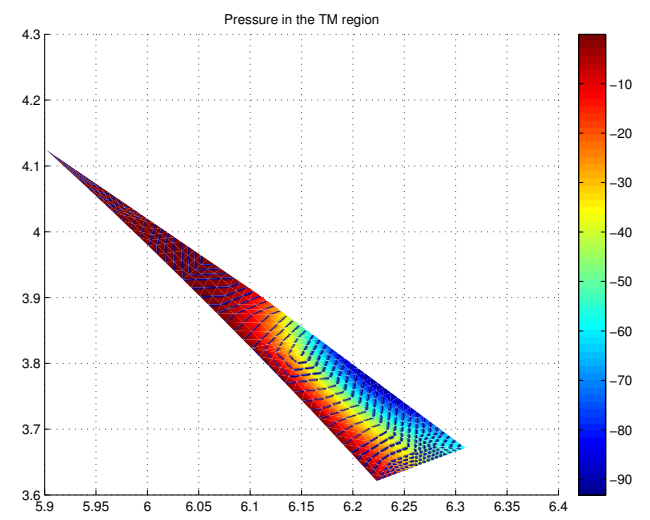

Figure 4.15: Computed pressure profile in the $\mathrm{TM}, h=1 / 4$, for a parabolic inflow and outflow profile.

[8] E. Burman and P. Hansbo. A unified stabilized method for Stokes' and Darcy's equations. J. Comput. Appl. Math., 198(1):35-51, 2007.

[9] Y. Cao, M. Gunzburger, X. Hu, F. Hua, X. Wang, and W. Zhao. Finite element approximations for Stokes-Darcy flow with Beavers-Joseph interface conditions. SIAM J. Numer. Anal., 47(6):4239-4256, 2010.

[10] M.A. Case, V.J. Ervin, A. Linke, and L.G. Rebholz. A connection between Scott-Vogelius and grad-div stabilized Taylor-Hood FE approximations of the Navier-Stokes equations. SIAM J. Numer. Anal., 49:1461-1481, 2011.

[11] A. Çeşmelioğlu and B. Rivière. Primal discontinuous Galerkin methods for time-dependent coupled surface and subsurface flow. J. Sci. Comput., 40(1-3):115-140, 2009. 
[12] M.W. Charles and N. Brown. Dimensions of the human eye relevant to radiation protection. Phys. Med. Biol., 20:202-218, 1975.

[13] T.S. Dietlein, P.C. Jacobi, C. Lűke, and G.K. Kreiglstein. Morphological variability of the trabecular meshwork in glaucoma patients: Implications for non-perforating glaucoma surgery. Br. J. Ophthalmol., 84:1354-1359, 2000.

[14] M. Discacciati, E. Miglio, and A. Quateroni. Mathematical and numerical models for coupling surface and groundwater flows. Appl. Numer. Math., 43:57-74, 2002.

[15] V.J. Ervin. Approximation of axisymmetric Darcy flow. Technical report, Dept. Math. Sci., Clemson University, 2012. http://www.clemson.edu/ces/math/technical_reports/ervin.axidarcy_drv.pdf.

[16] V.J. Ervin and E.W. Jenkins. Stenberg's sufficiency condition for axisymmetric Stokes flow. Technical report, Dept. Math. Sci., Clemson University, 2011. http://www.clemson.edu/ces/math/technical_reports/ervin.axisym_sten.pdf.

[17] V.J. Ervin, E.W. Jenkins, and S. Sun. Coupled generalized non-linear Stokes flow with flow through a porous media. SIAM J. Numer. Anal., 47:929-952, 2009.

[18] L. Formaggia, J.-F. Gerbeau, F. Nobile, and A. Quateroni. Numerical treatment of defective boundary conditions for the Navier-Stokes equations. SIAM J. Numer. Anal., 40:376-401, 2002.

[19] J. Galvis and M. Sarkis. Non-matching mortar discretization analysis for the coupling StokesDarcy equations. Electron. Trans. Numer. Anal., 26:350-384, 2007.

[20] G.N. Gatica, R. Oyarzúa, and F.-J. Sayas. Analysis of fully-mixed finite element methods for the Stokes-Darcy coupled problem. Math. Comp., 80(276):1911-1948, 2011.

[21] R.H.W. Hoppe, P. Porta, and Y. Vassilevski. Computational issues related to iterative coupling of subsurface and channel flows. Calcolo, 44:1-20, 2007.

[22] O. Iliev and V. Laptev. On numerical simulation of flow through oil filters. Computing and Visualization in Science, 6:139-146, 2004.

[23] W. Jäger and A. Mikelić. On the interface boundary condition of Beavers, Joseph, and Saffman. SIAM J. Appl. Math., 60:1111-1127, 2000.

[24] M. Johnson. What controls aqueous humor outflow resistance? Exp. Eye Res., 82:545-557, 2006.

[25] H. Kolb. Gross anatomy of the eye. at http://webvision.med.utah.edu/book/part-ifoundations/gross-anatomy-of-the-ey/.

[26] W. Layton, C. Manica, M. Neda, M.A. Olshanskii, and L. Rebholz. On the accuracy of the rotation form in simulations of the Navier-Stokes equations. J. Comput. Phys., 228(5):3433$3447,2009$.

[27] W.J. Layton, F. Schieweck, and I. Yotov. Coupling fluid flow with porous media flow. SIAM J. Numer. Anal., 40:2195-2218, 2003. 
[28] Y.-J. Lee and H. Li. On stability, accuracy, and fast solvers for finite element approximations of the axisymmetric Stokes problem by Hood-Taylor elements. SIAM J. Numer. Anal., 49(2):668691, 2011.

[29] H. Li. Finite element analysis for the axisymmetric Laplace operator on polygonal domains. $J$. Comput. Appl. Math., 235(17):5155-5176, 2011.

[30] M. Mu and J. Xu. A two-grid method of a mixed Stokes-Darcy model for coupling fluid flow with porous media flow. SIAM J. Numer. Anal., 45(5):1801-1813, 2007.

[31] F.W. Newell. Ophthalmology: Principles and Concepts, volume 15 of Texts in Applied Mathematics. Mosby, St. Louis, eighth edition, 1996.

[32] M. Olshanskii, G. Lube, T. Heister, and J. Löwe. Grad-div stabilization and subgrid pressure models for the incompressible Navier-Stokes equations. Comp. Meth. Appl. Mech. Eng., 198:3975-3988, 2009.

[33] M.A. Olshanskii. A low order Galerkin finite element method for the Navier-Stokes equations of steady incompressible flow: A stabilization issue and iterative methods. Comp. Meth. Appl. Mech. Eng., 191:5515-5536, 2002.

[34] M.A. Olshanskii and A. Reusken. Grad-Div stabilization for the Stokes equations. Math. Comp., 73:1699-1718, 2004.

[35] International Commission on Radiological Protection. Task Group on Reference Man. Report of the Task Group on Reference Man. ICRP No. 23. Pergamon Press, Oxford; New York, 1975.

[36] P.-A. Raviart and J. M. Thomas. Dual finite element models for second order elliptic problems. In Energy methods in finite element analysis, pages 175-191. Wiley, Chichester, 1979.

[37] B. Riviére and I. Yotov. Locally conservative coupling of Stokes and Darcy flows. SIAM J. Numer. Anal., 42:1959-1977, 2005.

[38] P. Saffman. On the boundary condition at the surface of a porous media. Stud. Appl. Math., 50:93-101, 1971. 